\title{
ANOMALIES AND SCHWINGER TERMS IN NCG FIELD THEORY MODELS
}

\author{
E. Langmann, J. Mickelsson, And S. Rydh
}

Theoretical Physics, Royal Institute of Technology, SE-10044, Stockholm, Sweden

March 1, 2001

\begin{abstract}
We study the quantization of chiral fermions coupled to generalized Dirac operators arising in NCG Yang-Mills theory. The cocycles describing chiral symmetry breaking are calculated. In particular, we introduce a generalized locality principle for the cocycles. Local cocycles are by definition expressions which can be written as generalized traces of operator commutators. In the case of pseudodifferential operators, these traces lead in fact to integrals of ordinary local de Rham forms. As an application of the general ideas we discuss the case of noncommutative tori. We also develop a gerbe theoretic approach to the chiral anomaly in hamiltonian quantization of NCG field theory.
\end{abstract}

\section{INTRODUCTION}

The nonabelian chiral anomaly appears in two different (but related) ways. First, it modifies the structure of current algebra. Historically, this was the way quantum mechanical symmetry breaking was first observed, [1], but it took some time before a clear mathematical formulation of this phenomenon was obtained, [2]. On the other hand, the anomaly appears in the path integral formulation as a lack of gauge invariance of the effective action, [3]. In either way, the anomaly can be thought of as an element of an appropriate group (or Lie algebra) cohomology group.

The nonabelian gauge anomaly arises from a left-right nonsymmetric coupling of vector potentials to a Dirac fermion field. This process can be generalized in a straight-forward way in noncommutative geometry models. Instead of a standard Dirac operator one considers self-adjoint (invertible) unbounded operators $D$ with the property that $1 /|D|^{p}$ is 'almost' trace-class for a given real number $p \geq 1$. 'Almost' means that the spectral integral $\int_{0}^{\Lambda}\left(|\lambda|^{p}+\epsilon\right)^{-1} d \mu(\lambda)$ is at most logarithmically divergent at $\Lambda \rightarrow \infty$, for any positive number $\epsilon$. After fixing such an operator we

Typeset by $\mathcal{A} \mathcal{M S}-\mathrm{T}_{\mathrm{E}} \mathrm{X}$ 
study generalized Dirac operators $D_{A}=D+A$ where $A$ is any hermitean bounded perturbation.

We stress that our considerations are very general: The family of Dirac operators could for example arise from a coupling of vector potentials through a star product (generalized Moyal brackets). All what is needed is that the $L_{p}$ estimate mentioned above is valid. This has been proven to hold in a class of star product quantizations [4] defined by a constant antisymmetric $\theta$ matrix. We shall study some consequences of this in section 7 .

Our starting point for a construction of a NCG field theory model is a triple $\left(D, \int, \mathcal{B}\right)$, where $D$ is the Dirac operator acting in a Hilbert space $H, \mathcal{B}$ is an associative algebra of operators in $H$ such that $[D, b]$ is bounded for all $b \in \mathcal{B}$. In addition, there is an 'integration', or a generalized trace map, from operators ('p-forms') of the type $\omega=b_{0}\left[\epsilon, b_{1}\right]\left[\epsilon, b_{2}\right] \ldots\left[\epsilon, b_{p}\right]$, or alternatively $|D|^{-p} b_{0}\left[D, b_{1}\right] \ldots\left[D, b_{p}\right]$, to complex numbers, [5], see also the discussion around Definition 10.8 in [24] for more details. Here $\epsilon=D /|D|$ is the sign of the Dirac operator. In some cases (but not always) one can prove an equality between the integrals of the two alternative expressions, [5]. The generalized vector potentials are then linear combinations of 1-forms $A=b_{0}\left[D, b_{1}\right]$, or sometimes $a=b_{0}\left[\epsilon, b_{1}\right]$.

We study the BRST double complex based on de Rham forms on the space of vector potentials $A$ with values in the space of the above operator p-forms $\omega$, with $p=0,1,2, \ldots$ The NCG BSRT complex has been previously studied from different points of view in [6] and more recently in [7].

One of the central results in the present paper concerns the question of locality of the BRST cocycles. In abstract NCG models it is not a priori clear what this could mean because in the operator approach there is in general no space-time manifold to define local fields. However, in the ordinary space-time geometric setup many of the basic quantities can be written as 'traces' of commutators of pseudodifferential operators. For example, this holds for nonabelian Schwinger terms [8] and the gauge anomaly in the path integral formulation [9]. It turns out that these traces are in fact integrals of local differential forms. We adopt this as our starting point: We prove that in the NCG field theory models there is a 'local' anomaly formula expressing the BRST cocycles as traces of commutators of nontrace-class operators.

The second main result concerns the description of the hamiltonian anomaly in gerbe theoretic terms in which the basic object is a 3-cohomology class, the Dixmier-Douady class. The results are largely generalizations of the corresponding considerations for the standard coupling of local vector potentials to chiral fermions [10]. However, there is one important technicality which makes a difference between the 'classical' and the NCG case. In the classical case the Dixmier-Douady class defines a de Rham form on the space of gauge orbits $\mathcal{A} / \mathcal{G}$. This is a smooth manifold when one restricts to the case of so-called based gauge transformations $\mathcal{G}_{0}$. In the NCG models the concept of based gauge transformations is not well-defined and the space of gauge orbits has singularities.

In order to avoid these singularities we realize that space of NCG gauge potentials for the Schatten index $p$ as loops in gauge potentials for index $p-1$. Correspondingly, 
the group of gauge transformations for index $p$ is the group of smooth loops in the gauge group for the case of index $p-1$ which allows us to define based gauge transformations in the usual way as loops pass the neutral element at a fixed value of the argument. This leads then to a construction of a gerbe and its DixmierDouady class as a de Rham form in a standard way.

We want to thank Göran Lindblad for drawing to our attention the reference $[23]$.

\section{THE GENERAL SETUP FOR NCG DESCENT EQUATIONS AND ANOM- ALIES}

Let $D_{0}$ be an unbounded selfadjoint operator in a complex Hilbert space $H$ such that $\left|D_{0}\right|^{-1} \in L_{p+}$, that is, $\left|D_{0}\right|^{-p} \in L_{1+}$ for some $p \geq 1$. Here $L_{1+}$ is the Dixmier ideal in the algebra of bounded operators in $H$. A positive operator $T$ is in $L_{1+}$ if it is compact and

$$
\frac{1}{\log N} \sum_{k=1}^{N} \lambda_{k}
$$

has a finite limit, where $\lambda_{1} \geq \lambda_{2} \geq \ldots$ are the eigenvalues of $T$. We also assume for simplicity that $D_{0}$ is invertible. However, the following discussion can be easily generalized to the case when $D_{0}$ is a finite rank perturbation of an invertible operator.

We shall work with bounded perturbations of the 'free Dirac operator' $D_{0}$, denote $D_{A}=D_{0}+A$ where $A$ is a bounded selfadjoint operator in $H$ such that $\left[\left|D_{0}\right|, A\right]$ is bounded. We shall denote $F_{A}$ a smoothed sign operator associated to $D_{A}$. The technical complication is that the map $A \mapsto D_{A} /\left|D_{A}\right|$ is not continuous when $D_{A}$ has zero modes. Instead, we can take a smooth function $f: \mathbb{R} \rightarrow \mathbb{R}_{+}$such that $f(x)-|x|$ approaches zero faster than any power of $x$ as $|x| \mapsto \infty$ and $f(x) \geq m$ for some positive constant, and we define $F_{A}=D_{A} / f\left(D_{A}\right)$. For example, take $f(x)=+\sqrt{x^{2}+e^{-x^{2}}}$. Then $A \mapsto F_{A}$ is norm continuous. If $D_{A}$ is the classical Dirac operator associated to a vector potential $A$ on a compact manifold then the difference $D_{A} /\left|D_{A}\right|-F_{A}$ is an infinitely smoothing pseudodifferential operator and in particular a trace class operator.

The sign operator $D_{A} /\left|D_{A}\right|$ defines a Fock space representation of the canonical anticommutation relations algebra CAR. The generators in the CAR algebra are denoted by $a(v), a^{*}(v)$, where $v \in H$. The algebra is defined by the basic relations

$$
\begin{aligned}
a(u) a(v)+a(v) a(u) & =0=a^{*}(u) a^{*}(v)+a^{*}(v) a^{*}(u) \\
a^{*}(u) a(v)+a(v) a^{*}(u) & =<v, u>,
\end{aligned}
$$

where the Hilbert space inner product $<., .>$ is antilinear in the first argument. The Dirac representation is then fixed by the requirement that there is a vacuum 
vector $\mid A>\in \mathcal{F}_{A}$ such that

$$
a^{*}(u)|A>=0=a(v)| A>\text { for } u \in H_{-}(A) \text { and } v \in H_{+}(A)
$$

where $H=H_{+}(A) \oplus H_{-}(A)$ is the polarization to the spectral subspaces $D_{A} \geq 0$, $D_{A}<0$, respectively.

Because of the potential zero modes of the Dirac operator the Dirac vacuum cannot in general be defined as a continuous function of $A$. Instead, we must be content with a choice of a polarization $H=W(A) \oplus W(A)^{\perp}$ such that $W(A) \sim H_{+}(A)$, where the equivalence is defined by the condition that the projection operators to the given subspaces differ only by Hilbert-Schmidt operators. The Hilbert-Schmidt condition comes from the requirement that the CAR representations defined by the two polarizations should be equivalent; for a review on CAR representations see [11]. Let $\epsilon_{W}=\pi_{W}-\pi_{W \perp}$ the grading operator defined by the orthogonal projection $\pi_{W}: H \rightarrow W$. Then $\epsilon_{W(A)}-\epsilon_{H_{+}(A)}$ is Hilbert-Schmidt and $\epsilon_{H_{+}(A)}-F_{A}$ is trace class and so $F_{A}$ is an approximation of $\epsilon_{W(A)}$ modulo Hilbert-Schmidt operators. The advantage of working with $F_{A}$ is that it is easier to produce explicit formulas (as we saw above), compared with the grading operators $\epsilon_{W(A)}$.

As an operator function $D_{A} / f\left(D_{A}\right)$ of $D_{A}$, the operator $F_{A}$ satisfies $g^{-1} F_{A} g=$ $F_{A^{g}}$, with $A^{g}=g^{-1} A g+g^{-1}\left[D_{0}, g\right]$ for a unitary transformation $g$ such that $\left[D_{0}, g\right]$ is bounded. Denote by $\mathcal{B}$ the algebra of bounded operators $b$ in $H$ such that $\left[D_{0}, b\right]$ and $\left[\left|D_{0}\right|, b\right]$ are bounded. Then all the operators $A=b_{0}\left[D_{0}, b_{1}\right]$, for $b_{i} \in \mathcal{B}$, satisfy the condition $\left[\left|D_{0}\right|, A\right]$ is bounded. We denote by $U_{p+}$ the group of unitary elements in $\mathcal{B}$. Any element $g \in U_{p+}$ satisfies $[\epsilon, g] \in L_{p+}$ where $\epsilon=D_{0} /\left|D_{0}\right|$.

Lemma 2.3. $F_{A}-\epsilon \in L_{p+}$ for any bounded operator $A$ such that $\left[\left|D_{0}\right|, A\right]$ is bounded.

Proof. 1) We first prove that $\left|D_{0}+A\right|-\left|D_{0}\right|$ is bounded. We have assumed that $D_{0}$ is invertible, so $\left|D_{0}\right| \geq \mu$ for some positive constant $\mu$. We use the norm estimate ||$|X|-|Y||| \leq \||| X^{*} X-\left.Y^{*} Y\right|^{1 / 2}||$, see for example [23, Section X.2]. Set $G=D_{0} A+$ $A D_{0}$ and split $G=G_{+}+G_{-}$where $G_{+}$commutes with $\epsilon$ and $G_{-}$anticommutes with $\epsilon$. The linear equation

$$
G=\left|D_{0}\right| Z+Z\left|D_{0}\right|
$$

can be solved as $Z=Z_{+}+Z_{-}$, with $Z_{+}=A_{+} \epsilon$ and

$$
Z_{-}=\int_{0}^{\infty} e^{-t\left|D_{0}\right|}\left[\left|D_{0}\right|, \epsilon A_{-}\right] e^{-t\left|D_{0}\right|} d t
$$

and thus $Z$ is bounded, since $\left\|e^{-t\left|D_{0}\right|}\right\| \leq e^{-t \mu}$ and since $\left[\left|D_{0}\right|, \epsilon A_{-}\right]=\epsilon\left[\left|D_{0}\right|, A_{-}\right]$ is bounded by assumption. Setting $X=D_{0} A$ and $Y=\left|D_{0}\right|+Z$ in the above norm estimate we observe that $\left|D_{0}+A\right|-|| D_{0}|+Z|$ is bounded. In the case when $\|Z\| \leq \mu$ the operator $\left|D_{0}\right|+Z$ is positive and so ||$D_{0}|+Z|=\left|D_{0}\right|+Z$ and it follows that $\left|D_{0}+A\right|-\left|D_{0}\right|=\left(\left|D_{0}+A\right|-|| D_{0}|+Z|\right)+\left(|| D_{0}|+Z|-\left|D_{0}\right|\right)$ is bounded. In the 
general case, replace $D_{0} \mapsto D^{\prime}=D_{0}+i \alpha$ for a real number $\alpha$. Clearly $\left|D_{+} i \alpha\right|-\left|D_{0}\right|$ and $\left|D_{0}+A+i \alpha\right|-\left|D_{0}+A\right|$ are bounded, so the question whether $\left|D_{0}+A\right|-\left|D_{0}\right|$ is bounded is equivalent to whether $\left|D^{\prime}+A\right|-\left|D^{\prime}\right|$ is bounded. Now $\left|D^{\prime}\right| \geq|\alpha|$ and so we have $\|Z\| \geq\left|D^{\prime}\right|$ when we choose $\alpha$ large enough and the proof reduces to the special case above.

2) We may assume without essential restriction that $D_{0}+A$ is invertible so we can select $f(x)=|x|$ and

$$
\begin{aligned}
F_{A}-\epsilon & =\left(D_{0}+A\right)^{-1}\left|D_{0}+A\right|-D_{0}^{-1}\left|D_{0}\right|=\left(1+D_{0}^{-1} A\right)^{-1} D_{0}^{-1}\left|D_{0}+A\right|-D_{0}^{-1}\left|D_{0}\right| \\
& =\left\{\left(1+D_{0}^{-1} A\right)^{-1}-1\right\} \epsilon+\left(1+D_{0}^{-1} A\right)^{-1} D_{0}^{-1} T,
\end{aligned}
$$

for some bounded operator $T$. The second term on the right is then in $L_{p+}$ since $D_{0}^{-1} \in L_{p+}$ and the first term is of the form $\left((1+S)^{-1}-1\right) \epsilon$ with $S \in L_{p+}$ and thus it belongs also to $L_{p+}$.

The following discussion is based on a parameterization of the fermionic Fock space by the operator $a=F_{A}-\epsilon$. Thus the Dirac vacuum for the vector potential $A$ is given by any grading operator $\epsilon_{a}$ which differs from $\epsilon+a$ by a HilbertSchmidt operator. Geometrically, this leads to the construction of a smooth infinitedimensional vector bundle $\mathcal{F}$ over the parameter space $\mathcal{A}$ of generalized vector potentials. The fiber $\mathcal{F}_{a}$ is the Fock space defined by the grading operator $\epsilon_{a}$. The quantized Dirac operator $\hat{D}_{A}$ acts as an unbounded positive operator in the fiber.

The group $U_{p+}$ acts in the base space of the bundle. The problem arises whether the action can be lifted to the total space such that $g^{-1} \hat{D}_{A} g=\hat{D}_{A^{g}}$. In case of smooth vector potentials, $A=\gamma^{k} A_{k}$, and massless Dirac operator it is known that the answer is negative. Instead, there is an extension $\hat{\mathcal{G}}$ of the group of gauge transformations $\mathcal{G}$ which acts in the total space. The obstruction to the $\mathcal{G}$ action is the extension term in the commutation relations of the Lie algebra $\operatorname{Lie}(\mathcal{G})$. This extension (Schwinger term) is a 2-cocycle $c_{n, 2}$ of $\operatorname{Lie}(\mathcal{G})$ in the module of complex functions of the variable $A$. In the case of one space dimension, $n=1$, the cocycle does not depend on $A$ and one may restrict to the module of constant functions.

There is an equivalent alternative way to view the obstruction. The standard construction in canonical quantization leads naturally to a bundle $P \mathcal{F}$ of projective Fock spaces which do not depend on any choices of the grading operator $\epsilon_{a}$. The existence of a Fock bundle which gives $P \mathcal{F}$ as its projectivization is then related to triviality of a Dixmier-Douady class in $H^{3}(\mathcal{A} / \mathcal{G}, \mathbb{Z}),[10]$.

The main content of the present article is to explain how the local formulas for gauge anomalies, Schwinger terms, the Dixmier-Douady class, and all the cocycles related to these through the standard BRST descent equations extend to certain cocycles on the space of bounded operators $A$ and the Lie algebra $\operatorname{Lie}\left(U_{p+}\right)$ such that a restriction to the classical case gives standard local formulas. A central ingredient is to use (noncyclic) extensions of the trace functional to nontrace-class operators. 
The 'infinitesimal version' of the gauge transformation $A \mapsto A^{g}$ in terms of the parameter $a$ is

$$
\delta_{X} a=[a, X]+[\epsilon, X] \text { for } X \in \mathbf{u}_{p}=\operatorname{Lie}\left(U_{p+}\right) .
$$

Let us recall the basic definitions in NCG differential calculus for Fredholm modules [5]. The differentials of order $n$ are linear combinations of operators of the type $b_{0}\left[\epsilon, b_{1}\right] \ldots\left[\epsilon, b_{n}\right]$ where $b_{i} \in \mathcal{B}$. One denotes $d b=[\epsilon, b]$ for $b \in \mathcal{B}$. If $\phi \in \Omega^{n}$ is a differential of order $n$ then $d \phi=\epsilon \phi+(-1)^{n+1} \phi \epsilon$. This gives a map $d: \Omega^{n} \rightarrow \Omega^{n+1}$ with $d^{2}=0$. The cohomology of this complex is trivial.

The coboundary operator associated to infinitesimal gauge transformations is denoted by $\delta$. We work with cochains of order $k, \tau \in \Omega_{k}$, consisting of functions $\tau\left(a ; X_{1}, \ldots, X_{k}\right)$ of $a \in \Omega^{1}$ and of Lie algebra elements $X_{i} \in \mathbf{u}_{p}$, linear in each $X_{i}$ and totally antisymmetric in the arguments $X_{i}$. The standard Lie algebra coboundary operator is defined by

$$
\begin{aligned}
\left(\delta \tau_{n}\right)\left(a ; X_{1}, \ldots, X_{n+1}\right) & =\sum_{i}(-1)^{i-1} \delta_{X_{i}} \tau\left(a ; X_{1}, \ldots, \hat{X}_{i}, \ldots, X_{n+1}\right)+ \\
& \sum_{i<j}(-1)^{i+j} \tau\left(a ;\left[X_{i}, X_{j}\right], . ., \hat{X}_{i}, \ldots, \hat{X}_{j}, \ldots, X_{n+1}\right),
\end{aligned}
$$

where the hat means that the corresponding argument is deleted and $\delta_{X_{i}}$ is the Lie derivative acting on functions of $a$, the action on the argument being given by (2.4). We remind that the multilinear forms $\tau$ on a Lie algebra can be interpreted as left invariant differential forms on the corresponding Lie group (and vice versa) through the standard identification of a Lie algebra as left invariant vector fields.

We shall also work with a $(d, \delta)$ double complex consisting of $\delta$ forms taking values in the $d$ complex $\Omega^{*}$, using the standard BRST sign convention. The BRST ghost $v$ can be interpreted as the Maurer-Cartan 1-form on the gauge group, that is, at the identity element it is the the tautological 1-form sending a Lie algebra element onto itself, $v=g^{-1} \delta g$. The sign conventions are encoded into the algebraic rules

$$
\begin{aligned}
d^{2} & =\delta^{2}=\delta d+d \delta=0 \\
\delta(v) & =-v^{2}=\delta v+v \delta \\
\delta(a) & =-[a, v]_{+}-v^{2}=\delta a+a \delta \\
d(v) & =[\epsilon, v]_{+}=d v+v d \\
d(a) & =[\epsilon, a]_{+}=d a+a d .
\end{aligned}
$$

We denote $[a, b]_{+}=a b+b a$.

Here a remark on notation is in order. We want to treat at the same time both the even and odd Fredholm modules (related to odd/even $k$.) To obtain the correct signs in (2.6), we need to make a reinterpretation of $x=v, \delta, \epsilon$ and $a$. 
Even Fredholm module. In this case we have, by definition, a hermitean operator $\Gamma$ in $H$ with $\Gamma^{2}=1$ which anticommutes with $\epsilon$ and $a$, and the correct signs are accounted for if we interpret the ghost as $\Gamma v$. To be precise, one should distinguish between $v, \delta, \epsilon$ and $a$ and

$$
\begin{aligned}
& s(v)=\Gamma v, \quad s(\delta)=\Gamma \delta . \\
& s(\epsilon)=\epsilon, \quad s(a)=a .
\end{aligned}
$$

Odd Fredholm module. We do not have a $\Gamma$ at hand but we introduce it by doubling the original Hilbert space, $H \mapsto H \otimes \mathbb{C}^{2}$, and introducing the usual Pauli sigma matrices $\sigma_{3}$ and $\sigma_{1}$ acting on the second factor. We can then define

$$
\begin{aligned}
& s(v)=v \otimes \sigma_{1}, \quad s(\delta)=\delta \otimes \sigma_{1} \\
& s(\epsilon)=\epsilon \otimes \sigma_{3}, \quad s(a)=a \otimes \sigma_{3} .
\end{aligned}
$$

In particular, the formulas $d(v)=[\epsilon, v]_{+}$and $d(a)=[\epsilon, a]_{+}$mean

$$
\begin{aligned}
& d(s(v))=[s(\epsilon), s(v)]_{+}=[\epsilon, v] \otimes \sigma_{3} \sigma_{1} \\
& d(s(a))=[s(\epsilon), s(a)]_{+}=[\epsilon, a]_{+} \otimes \sigma_{3}^{2}=[\epsilon, a]_{+} \otimes 1 .
\end{aligned}
$$

where the sigma matrices account for the correct signs. We stress that the auxiliary space is just a tool to keep track of the signs (the even and odd case could actually be handled in a unified manner, but we choose to utilize $\Gamma$ in the even case).

In both cases, the symbols $x=v, \delta, \epsilon$ and $a$ in (2.6) should actually be interpreted as $s(x)$ as specified above, and only for simplicity of the notation we write $x$ instead of $s(x)$. We will sometimes also use this simplified notation below, in particular in the Appendix, but we will always clearly point this out.

In the standard discussion of anomalies in quantum field theory one constructs cocycles $c_{n, k}$ in the $\left(\Omega_{*}, \delta\right)$ complex by integrating de Rham forms $\omega_{n, k}$ in $\Omega_{*}^{n}$ over a compact manifold of dimension $n$. In the NCG setting integration of forms is replaced by applying an appropriate trace functional to the operator valued forms. In fact, in the case of an odd Fredholm module the integral is normally defined as the trace $\operatorname{tr}_{C} \phi$ where the conditional trace is defined as $\operatorname{tr}_{C}(X)=\frac{1}{2} \operatorname{tr}(X+\epsilon X \epsilon)$. However, in our notation we have to take the trace also in the auxiliary space $\mathbb{C}^{2}$, and the correct definition is

$$
\int \phi=\frac{1}{2} \operatorname{tr}_{C} \sigma_{3} \phi
$$

In the case of an even Fredholm module the standard definition of the integral is $\operatorname{tr}_{C}(\Gamma \phi)$ whereas with our conventions the operator expression $\phi$ is already equipped (for odd $k$ ) with the $\Gamma$ factor and we set

$$
\int \phi=\operatorname{tr}_{C} \phi .
$$


In the case of even $k$ (in an even module) the integral vanishes.

The translation from the classical to NCG setting is straight-forward. The de Rham exterior derivation is replaced by the operation $d$ described above (to the forms after the symbol ' $\int$ '). All the formal manipulations are done exactly in the same way as in the classical BRST complex. Of course, the nontriviality of the cohomology classes depends on what is meant by the trace (and the definition of the trace is intertwined by the choice of $\left.\left(H, D_{0}, \mathcal{B}\right)\right)$.

The construction of a family of cocycles $c_{j, k}$ over a Fredholm module starts from the (NCG) operator valued Chern class $F^{n}$, where $F=d(a)+a^{2} \in \Omega^{2}$ is the curvature. The Chern-Simons form is defined by

$$
c_{2 n-1,0}(a)=\int n \int_{0}^{1} d t a\left[t d(a)+t^{2} a^{2}\right]^{n-1}
$$

where $t d(a)+t^{2} a^{2}=F(t)$ is the curvature associated with $t a$ (a path connecting $a$ and 0 ). The other terms in the complex (starting from the Chern class) are given by similar formulas where one has the curvature associated with $d+\delta$ and a path of vector potentials connecting $a+v$ and 0 ,

$$
c_{2 n-k-1, k}=\left.\int n \int_{0}^{1} d t\left(a\left[t d(a)+t^{2} a^{2}+(1-t) d(v)\right]^{n-1}\right)\right|_{[k]}, \quad k=0, \ldots, n-1
$$

where now the curvature in the square brackets is obtained starting from the vector potential $t a+v$, and $\left.(\cdots)\right|_{[k]}$ means the projection onto those terms which are of degree $k$ in the 'ghost' $v$, and similarly,

$$
c_{2 n-k-1, k}=\left.\int n \int_{0}^{1} d t\left(v\left[t d(v)+\left(t^{2}-t\right) v^{2}\right]^{n-1}\right)\right|_{[k]}, \quad k=n, \ldots, 2 n
$$

where now the curvature is associated with $t v$. We stress that in the previous formulas for $c_{2 n-k-1, k}$, we use the simplified notation where $x=v, a$ is short for $s(x)$ as discussed above. (We also recall that one can obtain different but equivalent formulas for $c_{2 n-k-1, k}$ depending on the choice of path $a+v \rightarrow 0$, and we find it convenient to use the path consisting of two straight lines $a+v \rightarrow v$ and $v \rightarrow 0$ leading to cocycles with lowest possible powers of $a[12]$.)

The crucial property are the cocycle relations $\delta c_{j, k}=0$ for all $j$ and $k$. In the standard case these are a consequence of the fact that the cocycles are integrals of traces of matrix valued de Rham forms, $c_{j, k}=\int_{M} \operatorname{tr} \omega_{j, k}$, which are linked by the so-called descent equations. These equations start with a relations connecting the Chern class and the Chern-Simons form, $F^{n}=d \omega_{2 n-1,0}+(\cdots)$, and then continue,

$$
\delta\left(\omega_{2 n-1-k, k}\right)+d\left(\omega_{2 n-1, k+1}\right)=(\cdots), \quad k=0, \ldots, 2 n-1
$$

where $(\cdots)$ are terms which have zero trace; they are explicitly commutators of matrix forms. Since all but the first term in the l.h.s. in these latter equations vanish 
under the combined trace and integral (the second term on the l.h.s. vanishes due to Stokes theorem) and $\delta \int=\int \delta$, one obtains the cocyle relations. In fact, in the standard case one usually applies to these descent equations the matrix trace, and therefore the terms $(\cdots)$ on the r.h.s. become zero. In the NCG generalizations, an analog of the separate matrix trace is usually not available (the matrix trace and the ordinary integration are combined in the abstract integral), and it is therefore natural to also keep the terms $(\cdots)[6]$. In fact, as we will show, the terms $(\cdots)$ contain interesting information: in case of the double complex based in a spectral triple they allow to connect the forms $\omega_{j, k}$ and $\omega_{j+2, k}$ in different dimensions $(d$ degree) but identical form degree, which, for example, provides an important link of the Schwinger terms in different dimensions (Theorem A4) and, in general, will allow us to obtain local forms of the NCG cocyles (Theorem 3.1).

As an example, the second Chern form is in the classical case the matrix trace of $F^{2}$, where $F=d(A)+A^{2}$ is the curvature form associated to a connection $A=$ $A^{\mu} d x_{\mu}$. In general, one then can check by straightforward algebraic manipulations

$$
F^{2}=d\left(\omega_{3,0}\right)+\left[a, \tilde{\omega}_{3,0}\right]_{+}
$$

where $\omega_{3,0}=\frac{1}{2}[d(a), a]_{+}+\frac{2}{3} a^{3}$ is the three dimensional Chern-Simons form (before applying the trace functional) and $\tilde{\omega}_{3,0}=\frac{1}{3}[d(a), a]_{+}+\frac{1}{2} a^{3}$ is another 3 -form. Note that for standard de Rham forms, the second term vanishes under the matrix trace. If we apply this the relation in the Fredholm module setting we use $\int F^{2}=\operatorname{tr}_{C}\left(\Gamma F^{2}\right)$, and since $\Gamma[a, \tilde{\omega}]_{+}=[\Gamma a, \tilde{\omega}]$ the second term becomes a trace of a commutator which vanishes for appropriate $p$. Of course, in the classical case also the integral of the first term is zero in the case of a manifold without boundary. The other descent equations are, in the simplified notation where $x=a, v, \omega_{3-k, k}$ etc. is short for $s(x)$ as in Eqs. (2.7)-(2.8),

$$
\begin{aligned}
\delta\left(\omega_{3,0}\right)+d\left(\omega_{2,1}\right) & =-\frac{1}{3}\left[a, \omega_{2,1}\right]_{+}-\left[v, \omega_{3,0}\right]_{+} \\
\delta\left(\omega_{2,1}\right)+d\left(\omega_{1,2}\right) & =-\frac{2}{3}\left[a, \omega_{1,2}\right]_{+}-\left[v, \omega_{2,1}\right]_{+} \\
\delta\left(\omega_{1,2}\right)+d\left(\omega_{0,3}\right) & =-\left[v, \omega_{1,2}\right]_{+} \\
\delta\left(\omega_{0,3}\right) & =-\frac{1}{2}\left[v, \omega_{0,3}\right]_{+}
\end{aligned}
$$

where

$$
\omega_{2,1}=\frac{1}{2}[a, d(v)]_{+}, \quad \omega_{1,2}=\frac{1}{2}[d(v), v]_{+}, \quad \omega_{0,3}=-\frac{1}{3} v^{3},
$$

all of them can be checked by straightforward algebraic manipulations. As we have already said, the BRST ghost $v$ can be interpreted as the Maurer-Cartan form on a group manifold, and thus are to be evaluated along tangent vectors $X_{j}$ at the neutral element (i.e, Lie algebra elements). Moreover, in the end we are interested in integrals of the operator forms and we need the treat the cases of odd or even Fredholm modules separately, as discussed above. 
For example, the form $\omega_{1,2}$ when evaluated for Lie algebra elements $X, Y$ and integrated in an odd module of appropriate Schatten index $p$, gives

$$
\int \omega_{1,2}(X, Y)=\frac{1}{2} \operatorname{tr}_{C}\left([d(X), Y]_{+}-[d(Y), X]_{+}\right)
$$

where $d(X)=[\epsilon, X]$, and $\omega_{0,3}$ leads to

$$
\int \omega_{0,3}(X, Y, Z)=-\operatorname{tr}_{C}[[X, Y], Z]_{+}
$$

and $\omega_{2,1}$ in an even module (appropriate $p$ ) leads to

$$
\int \omega_{2,1}(X)=-\frac{1}{2} \operatorname{tr}_{C}[a, \Gamma d(X)]_{+}=\frac{1}{2} \operatorname{tr}_{C} \Gamma[a, d(X)]
$$

\section{3. 'LOCAL' NCG ANOMALIES AND SCHWINGER TERMS}

In the case of the classical BRST complex all the cocycles $c_{j, k}$ are given in terms of differential forms which are differential polynomials in variables $a, v$. The Fredholm module cocycles involve terms like $[\epsilon, v], \epsilon a+a \epsilon$, and therefore are nonlocal in nature; when evaluated using the symbol calculus of pseudodifferential operators they contain terms of arbitrary high order in the partial derivatives.

However, even in the case of the Fredholm module cocycles (for classical vector potentials and gauge transformations) the locality is preserved in a certain sense. Namely, it turns out that the cocycles $c_{j, k}$ are equivalent (in the BRST cohomology) to cocycles $c_{j, k}^{\prime}$ which can be written as renormalized traces of commutators of PSDO's. In the case of Schwinger terms this was observed in [8] and the same principle was applied to the calculation of the gauge anomaly for the chiral Dirac determinant in [9]. The trace of a commutator depends only on the term in the asymptotic expansion of a PSDO which has order equal to $-\operatorname{dim} M$, and for this reason one needs to take into account only a finite number of derivatives of the symbols (since each differentiation in a homogeneous term decreases the order by one). In this sense the trace of a commutator is a local expression. In a more general setup, beyond the PSDO calculus, we take this as a definition of locality: cocycles which are traces of commutators in the algebra are called local.

We set up the following assumptions. There is a complex linear functional TR on the algebra generated by $D_{0},\left|D_{0}\right|$ and $\mathcal{B}$ such that 1 ) it is equal to the ordinary trace for trace class operators, 2) it has the property that $\operatorname{TR}[A, B]=0$ when $A B, B A \in L_{1+}$, and 3 ) $\mathrm{TR}[\epsilon, W]=0$ (odd case), $\mathrm{TR}[\Gamma \epsilon, W]=0$ (even case), for bounded operators $W$, with $\epsilon=D_{0} /\left|D_{0}\right|$. 
Example 1 Set $p=1$ and consider the cocycle $c(X, Y)=\operatorname{tr}_{C} X[\epsilon, Y]$, for $X, Y \in \mathcal{B}$. Here everything is defined in the original Hilbert space $H$ and not in $H \otimes \mathbb{C}^{2}$. We can write

$$
\begin{aligned}
c_{1,2}(X, Y) & =\frac{1}{4} \operatorname{tr} \epsilon[\epsilon, X][\epsilon, Y]=\operatorname{tr}_{C} X[\epsilon, Y] \\
& =\frac{1}{2} \operatorname{TR} X[\epsilon, Y]=\frac{1}{2} \operatorname{TR}[X \epsilon, Y]-\frac{1}{2} \operatorname{TR} \epsilon[X, Y] .
\end{aligned}
$$

The last term is the coboundary of the cochain $\theta(X)=\frac{1}{2} \mathrm{TR} \epsilon X$ and therefore the class of $c_{1,2}$ is given by

$$
c_{l o c}(X, Y)=\frac{1}{2} \operatorname{TR}[X \epsilon, Y] .
$$

One can also check by a direct computation that $c_{l o c}$ is a cocycle.

$$
\begin{aligned}
2\left(\delta c_{l o c}\right)(X, Y, Z) & =\operatorname{TR}\{[[X, Y] \epsilon, Z]+c y c l .\} \\
& =\operatorname{TR}\{[[X, Y],[\epsilon, Z]]+c y c l .\} \\
& =\operatorname{TR}[\epsilon,[[X, Y], Z]]+\operatorname{TR}[Y,[[\epsilon, X], Z]]-\operatorname{TR}[X,[[\epsilon, Y], Z]]+c y c l .
\end{aligned}
$$

By 3) the first term on the right vanishes and by 2) the second and third term vanish. In the case when $X, Y$ are multiplication operators, by smooth functions, on the circle $S^{1}$ the local cocycle becomes the central term in an affine Lie algebra,

$$
c_{l o c}(X, Y)=\frac{1}{2 \pi i} \int_{S^{1}} \operatorname{tr} X d Y,
$$

where the trace under the integral sign is a finite-dimensional matrix trace for the matrix valued functions $X, Y$.

The cocycle $c_{1,2}$ (or $c_{l o c}$ ) arises in canonical quantization in the following way. To each pair of basis vectors $e_{i}, e_{j}$ in $H$ there corresponds an operator $\hat{e}_{i j}=a^{*}\left(e_{i}\right) a\left(e_{j}\right)$ in the Fock representation. We may label the basis vectors such that $e_{i} \in H_{+}$ for $i=0,1,2, \ldots$ and $e_{i} \in H_{-}$for $i=-1,-2, \ldots$, where $H_{ \pm}=\frac{1}{2}(1 \pm \epsilon) H$. Then a matrix $\left(\alpha_{i j}\right)$ in this basis has the canonical quantization as the operator $\hat{\alpha}=\sum \alpha_{i j} \hat{e}_{i j}$. For infinite matrices this might diverge. Actually, that happens already when $\alpha$ is the unit matrix. To circumvent this one introduces the normal ordering $\hat{e}_{i j} \mapsto \hat{e}_{i j}-\delta_{i j} \theta(-i)$ with $\theta(x)=x$ for $x \geq 0$ and $\theta(x)=0$ for $x<0$. With these new operators $\hat{e}_{i j}$ the operator $\hat{\alpha}$ is defined in a dense domain for any bounded operator $\alpha$ such that $[\epsilon, \alpha]$ is Hilbert-Schmidt and the commutation relations are given by

$$
[\hat{\alpha}, \hat{\beta}]=\widehat{[\alpha, \beta}]+c(\alpha, \beta)
$$

[21].

Example 2 Here we consider the problem arising from quantization of gauge currents in three space dimensions. Typically, $[\epsilon, X]$ is not Hilbert-Schmidt but it belongs to the ideal $L_{3+} \subset L_{4}$. For this reason the expression for the 2-cocycle in 
the previous example does not converge for 3-dimensional gauge currents. Instead, one has to introduce a renormalization of the 2-cocycle,

$$
c_{3,2}(a ; X, Y)=\frac{1}{8} \operatorname{tr}_{C} a[[\epsilon, X],[\epsilon, Y]]
$$

with $a=F_{A}-\epsilon$. One can check by a direct calculation that this is a cocycle in the sense that

$$
c_{3,2}(a ;[X, Y], Z)+\delta_{X} c_{3,2}(a ; Y, Z)+\text { cyclic perm. of } X, Y, Z=0
$$

Let next $\eta(a ; X)=\frac{1}{8} \mathrm{TR} \epsilon a[\epsilon, X]$. By a direct calculation one can check that $c_{3,2}=$ $\delta \eta+c_{l o c}$ where now

$$
8 c_{l o c}(a ; X, Y)=\operatorname{TR}[Y, \epsilon a[\epsilon, X]]-\operatorname{TR}[X, \epsilon a[\epsilon, Y]]+2 \operatorname{TR}[X \epsilon, Y]-2 \operatorname{TR}[Y \epsilon, X]
$$

is explicitly a generalized trace of a sum of commutators. Using the 'bare' BRST notation (without the auxiliary space $\mathbb{C}^{2}$ ) we can write $c_{l o c}=\frac{1}{4} \operatorname{TR}[v \epsilon, v]-\frac{1}{8} \operatorname{TR}[v, \epsilon a[\epsilon, v]]$.

In this example the canonical quantization of gauge currents is ill-defined even after normal ordering, precisely because $[\epsilon, X]$ is not Hilbert-Schmidt. However, there is an operator theoretic interpretation for second quantized $\hat{X}, \hat{Y}$. These are now generators for unitary transformations between Fock spaces carrying inequivalent representations of the CAR algebra. Geometrically, there is a bundle of Fock spaces parametrized by the external field $a$ and the gauge transformations act as unitary maps between the fibers, [22].

Example 3 As a final example we discuss the gauge anomaly in two space-time dimensions. Here we are in the even case and we have $\Gamma \epsilon=-\epsilon \Gamma$ and we consider gauge transformations $X$ which commute with $\Gamma$. Then $c_{2,1}(a ; X)=\operatorname{tr}_{C} \Gamma a[\epsilon, X]$ is a cocycle,

$$
\delta_{X} c_{2,1}(a ; Y)-\delta_{Y} c_{2,1}(a ; X)-c_{2,1}(a ;[X, Y])=0,
$$

using the fact that $\operatorname{tr}_{C}[\epsilon, \cdot]=0$. In this case

$$
c_{2,1}(a ; X)=\operatorname{TR}[\Gamma a \epsilon, X]+(\delta \eta)(a)
$$

where $\eta(a)=$ TR $\Gamma a \epsilon$. In BRST notation, for an even module, $c_{l o c}=\operatorname{TR}[a \epsilon, s(v)]$. We leave it as an exercise for the reader to show that when inserting $\epsilon=D_{0} /\left|D_{0}\right|$, $D_{0}=-i \sum_{k=1}^{2} \gamma^{k} \frac{\partial}{\partial x_{k}}, D_{A}=D_{0}+\sum \gamma^{k} A_{k}(x)$, and $a=D_{A} /\left|D_{A}\right|-\epsilon$ one obtains the standard formula for the nonabelian gauge anomaly in two space-time dimensions,

$$
c_{l o c}=\frac{1}{4 \pi} \int \operatorname{tr}\left(A_{1} \partial_{2} X-A_{2} \partial_{1} X\right)
$$

for a smooth infinitesimal gauge transformation $X$ of compact support.

In the general case we have the following result: 
Theorem 3.1. Let $n=1,2, \ldots$ and $k=0,1,2, \ldots$ and let the cocycle $c_{2 n-1-k, k}$ be computed from the descent equations in Section 2. Then for even $k$ the cohomology class $\left[c_{2 n-1-k, k}\right]$ is represented by a cocycle $c_{2 n-1-k, k}^{\text {loc }}$ which is a generalized trace of a sum of commutators; each commutator is a polynomial in the operators a, $\epsilon$, and $X_{j}$ (the latter operators correspond to the tangent vectors at which the ghosts where evaluated). In the case of odd $k$ one has to add a term proportional to a generalized trace of $\omega_{0, k}$.

This theorem is a reformulation of Theorems A4 and A5 in the Appendix, which also gives more explicit formulas.

\section{A MODEL FOR $\mathcal{A} / \mathcal{G}$}

When $\mathcal{A}$ is the space of classical smooth vector potentials on a compact manifold $M$ and $\mathcal{G}_{0}$ is the group of based smooth gauge transformations (based means that $g(p)=1$ at some given point $p \in M)$ then the quotient $X=\mathcal{A} / \mathcal{G}_{0}$ is a smooth infinite dimensional Banach manifold. If one tries to generalize this to the NCG setting, by replacing $\mathcal{A}$ by all bounded perturbations of the free Dirac operator and taking $\mathcal{G}$ as the group of unitaries in the algebra $\mathcal{B}$, one encounters the problem that there is no natural way to define what is meant by based gauge transformations; this leads to the difficulty that $X$ is not a manifold, it has a lot of singularities since at a generic point in $\mathcal{A}$ the action of $\mathcal{G}$ is not free. Here we shall construct a model for $\mathcal{A}$ and $\mathcal{G}$ such that the quotient will be free of singularities.

Our construction is essentially based on Bott periodicity. Recall that the inductive limit $U(\infty)$ of the group $S U(N)$ as $N \mapsto \infty$ has odd homotopy type: All its homotopy groups $\pi_{2 k+1} U(\infty)$ are isomorphic to $\mathbb{Z}$ whereas the even homotopy groups are trivial. For this reason the group of based gauge transformations $f: M \rightarrow U(N)$, in the limit $N \mapsto \infty$, for $M=S^{2 n}$ is homotopic to $U(\infty)$. In the odd dimensional case, $M=S^{2 n+1}$, the group of gauge transformations has even homotopy type: All the even homotopy groups are isomorphic to $\mathbb{Z}$ and the odd homotopy groups are trivial. Denoting the gauge group in the even case by $G$ (which has the homotopy type of $U(\infty)$ ) then the group of gauge transformations in the odd case is the group of based loops $\Omega G$. This latter group has the homotopy type of $U_{p}^{\prime}$ for any $p \geq 1$ where $U_{p}^{\prime}$ is defined in the following way: Let $\epsilon$ be a grading operator (it could be the sign of a Dirac operator) in a Hilbert space, with eigenvalues \pm 1 , both eigenspaces infinite dimensional. Set $U_{p}^{\prime}(H)=\left\{g \in U(H) \mid[\epsilon, g] \in L_{p}\right\}$. Here $U(H)$ is the (contractible) group of all unitaries in the Hilbert space $H$. Recall that $U_{p+}(H)$ is the group of unitary elements in the algebra $\mathcal{B}$. All elements $g$ in $U_{p+}(H)$ satisfy $[\epsilon, g] \in L_{p+}$.

Let now $D_{p}, p$ an even integer, be a hermitean operator in $H$ such that $1 /\left|D_{p}\right| \in$ $L_{p+}$ and let $\Gamma$ be a hermitean operator in $H$ such that $\Gamma^{2}=1$ and $\Gamma D_{p}=-D_{p} \Gamma$. Let $D_{p+1}=i \Gamma \frac{d}{d t}+D_{p}$. This operator is self-adjoint in an appropriate dense domain in the Hilbert space $\mathcal{H}=L^{2}\left(S^{1}, H\right)$ and has the property $1 /\left|D_{p+1}\right| \in L_{(p+1)+}$. 
A generalized vector potential is defined as a hermitean time dependent bounded operator $A(t)$ in $H$. The 'Dirac operator' coupled to $A(t)$ is then $D_{p+1}+A(t)$. The vector potential can be split as $A=A_{0}+A_{1}$, where $A_{0}$ commutes with $\Gamma$ (the 'time component' of $A$ ) and $A_{1}$ anticommutes with $\Gamma$ (this is the 'space component' of $A$ ). The time dependent gauge transformations are smooth functions $g(t)$ with values in the group $U_{p+}(H, \Gamma)$ of unitary operators $g \in U_{p+}(H)$ such that $[\Gamma, g]=0$. We can split $H=H_{1} \oplus H_{2}$ to eigenspaces of $\Gamma$ corresponding to eigenvalues \pm 1 . Since $g \in U_{p+}(H, \Gamma)$ commutes with $\Gamma$ we can write $g$ as a direct sum of linear operators $g_{i}: H_{i} \rightarrow H_{i}, i=1,2$.

The group $U_{p+}(H, \Gamma)$ is a subgroup of the group $U_{p+}^{\prime}(H, \Gamma)$ which consists of unitary operators $g$ such that $[\epsilon, g] \in L_{p+}$ and $\Gamma g=g \Gamma$. These conditions mean that $g=g_{1} \oplus g_{2}$ with $g_{2}-\epsilon g_{1} \epsilon \in L_{p+}$. Thus the group $U_{p+}^{\prime}$ is parametrized by pairs of unitary operators $\left(g_{2}, h\right)$ with $g_{2}$ an arbitrary unitary operator in $H_{2}$ and $h=g_{2}^{-1} \epsilon g_{1} \epsilon$ an unitary operator in $H_{2}$ such that $h-1 \in L_{p+}$; we denote the group of these elements by $U^{p+}\left(H_{2}\right)$. Thus by Kuiper's theorem $U_{p+}^{\prime}(H, \Gamma)$ is homotopy equivalent to $U^{p+}\left(H_{2}\right)$. A similar result holds for the subgroup $U_{p+} \subset U_{p+}^{\prime}$. In this case one chooses as parameters $g_{2}$ and $\tilde{h}=g_{2}^{-1} D_{+}^{-1} g_{1} D_{+}$and the conditions are as before. Here $D_{+}: H_{2} \rightarrow H_{1}$ is the restriction of $D_{p}$ to $H_{2}$. (If $D_{+}$has zero modes, replace $D_{+}^{-1}$ by $D_{-} /\left(D_{-} D_{+}+1\right)$.)

For each time dependent perturbation there is a unique (nonperiodic) gauge transformation $g(t)$ such that $g(0)=1$ and $A^{\prime}(t)=g^{-1} A g+g^{-1}\left[D_{p}, g\right]+i g^{-1} \partial_{t} g$ is the generalized temporal gauge, i.e., the even component $A_{0}^{\prime}=0$. It follows that the quotient $\mathcal{A} / \mathcal{G}_{0}$, where $\mathcal{G}_{0}$ is the group of periodic gauge transformations $g(t)$ with $g(0)=1$, is equal to the product $\mathcal{A}_{1} \times U_{p+}(H, \Gamma)$. Here $\mathcal{A}_{1}$ is the space of bounded operators $A$ in $H$ such that $\Gamma A=-\Gamma A$ and the coordinate $g \in U_{p+}(H, \Gamma)$ comes from the holonomy $g=g(2 \pi)$ around the circle.

Since $\mathcal{A}_{1}$ is an affine space, $\mathcal{A} / \mathcal{G}_{0}$ is homotopy equivalent to $U_{p+}(H, \Gamma)$.

We end this section by a remark on the homotopy type of the various groups involved. When the condition $[\epsilon, g] \in L_{p+}$ is replaced by $[\epsilon, g] \in L_{p}$ we obtain the group $U_{p}^{\prime}(H, \Gamma) \subset U_{p+}^{\prime}(H, \Gamma)$. Note that also $U_{p+} \subset U_{p+\alpha}^{\prime}$ for any $\alpha>0$. Similarly one can define $U^{p}$ with $U^{p} \subset U^{p+} \subset U^{p+\alpha}$. According to Palais [13], the groups $U^{p}$ for all $p \geq 1$ are homotopy equivalent with $U(\infty)$. Similarly $U_{p}^{\prime}(H)$ is homotopy equivalent to the group $U_{0}^{\prime}(H)$ of unitary operators $g$ such that $[\epsilon, g]$ is of finite rank. It is plausible that the same holds for the groups $U_{p+}$ and $U^{p+}$, but the proof in [13] cannot directly be applied to these cases. The topology of these groups is determined by a norm topology on the operator ideals $L_{p+}$.

The natural norm on $L_{p+}$ for $p>1$ is given as

$$
\|T\|_{p+}=\sup _{N \geq 1} N^{\frac{1}{p}-1} \sigma_{N}(T)
$$

where $\sigma_{N}(T)$ is the sum of the $N$ largest eigenvalues of $|T|$. In the case $p=1$ the factor $N^{\frac{1}{p}-1}$ is replaced by $(\log N)^{-1}$. 


\section{THE GERBE OVER $\mathcal{A} / \mathcal{G}_{0}$}

Each $A \in \mathcal{A}$ and $\lambda \notin \operatorname{Spec}\left(D_{A}\right)$ defines a fermionic Fock space $\mathcal{F}_{A, \lambda}$ with a Dirac vacuum $|A, \lambda\rangle$. To begin with, we have the polarization $\mathcal{H}=\mathcal{H}_{+}(A, \lambda) \oplus \mathcal{H}_{-}(A, \lambda)$ to a pair of infinite dimensional subspaces, defined by the spectral projections $D_{A}>\lambda$ and $D_{A}<\lambda$. Then the Fock space $\mathcal{F}_{A, \lambda}$ is generated by the algebra of creation and annihilation operators $a^{*}(u), a(u)$ with the relations (2.1) and the characterization of the vacuum $|A, \lambda\rangle$ as in (2.2).

The Fock spaces depend on the choice of the vacuum level $\lambda$. However, for $\lambda, \mu \notin \operatorname{Spec}\left(D_{A}\right)$ there is a natural projective isomorphism $\mathcal{F}_{A, \lambda} \equiv \mathcal{F}_{A, \mu}$. This construction is equivariant with respect to the gauge group action, leading to a projective bundle $P \mathcal{F}$ over $\mathcal{A} / \mathcal{G}_{0}$. The question is whether there exists a true vector bundle $\mathcal{F}$ over $X=\mathcal{A} / \mathcal{G}_{0}$ such that $P \mathcal{F}$ is the projectivization of $\mathcal{F}$.

In general, there is an obstruction to the existence of $\mathcal{F}$. The obstruction can be described in terms of an element $\omega$ in $H^{3}\left(\mathcal{A} / \mathcal{G}_{0}, \mathbb{Z}\right)$. This may or may not correspond to a nontrivial de Rham cohomology class. However, in the present setting there is a nontrivial obstruction as a de Rham form.

A more geometric way to describe the obstruction problem is to construct a family of local complex line bundles $D E T_{\lambda \lambda^{\prime}}$ over $\mathcal{U}_{l l^{\prime}}=\mathcal{U}_{l} \cap \mathcal{U}_{l^{\prime}}$ with $\mathcal{U}_{l}=\{A \in$ $\left.\mathcal{A} / \mathcal{G}_{0} \mid \lambda \notin \operatorname{Spec}\left(D_{A}\right)\right\}$. Here $D E T_{l l^{\prime}}$ is the top exterior power of the (finite dimensional) spectral subspace of $D_{A}$ corresponding to the open interval $\left(l, l^{\prime}\right)$ (with $\left.l<l^{\prime}\right)$. These line bundles have a set of natural isomorphisms

$$
D E T_{l l^{\prime}} \otimes D E T_{l^{\prime} l^{\prime \prime}}=D E T_{l l^{\prime \prime}}
$$

which give the relations needed to define a gerbe over $\mathcal{A} / \mathcal{G}_{0}$. The gerbe is trivial if there is a family of local line bundles $D E T_{l}$ over the open sets $\mathcal{U}_{l}$ such that $D E T_{l l^{\prime}}=D E T_{l}^{-1} \otimes D E T_{l^{\prime}}$. Physically, these latter bundles are the local fermionic vacuum bundles. The nontriviality of the gerbe is measured by the nontriviality of the Dixmier-Douady class $\omega$, [14].

The nontriviality of the obstruction as a de Rham form follows from the considerations in $[10,15]$. The setting in the latter paper was similar to the present case except that a smaller base space, $G_{1}$ of unitaries which differ from the identity by a trace class operator, was considered instead of $U_{p+}(H, \Gamma)$.

On the group $G_{1}$ the obstruction form is particularly simple. It is given as the left invariant form

$$
\omega(X, Y, Z)=\frac{i}{8 \pi^{2}} \operatorname{tr} X[Y, Z]
$$

where $X, Y, Z \in \operatorname{Lie}\left(G_{1}\right)$. This is normalized such that its integral over a fundamental 3-cycle in $U^{3}(H) \simeq U_{3}^{\prime}(H, \Gamma)$ is equal to one. As it stands, $\omega$ does not extend to $U_{p}^{\prime}(H, \Gamma)$ for $p>3$. Instead, we have to construct another representative for the cohomology class which extends to $U_{p}^{\prime}(H, \Gamma)$, and actually also to the larger group $U_{p+}(H, \Gamma)$. Since $U_{q+}(H, \Gamma) \subset U_{p}^{\prime}(H, \Gamma)$ for $q<p$, we get a normalized representative for the cohomology class also in the former group. 
We shall treat both the even and odd cases at the same time and $U_{p}^{\prime}$ stands for $U_{p}^{\prime}(H, \Gamma)$ in the case of an even Fredholm module and $U_{p}=U_{p}^{\prime}(H)$ in the odd case. In both cases we denote the left invariant Maurer-Cartan 1-form on the group by $g^{-1} \delta g$.

Next we consider the $(d, \delta)$ BRST bicomplex on the group manifold $U_{p}^{\prime}$. As before, $\delta$ is the exterior differentiation on the group manifold with a choice of signs when acting on $d$ forms such that $d \delta+\delta d=0$. We set $\Delta=d+\delta$, so $g^{-1} \Delta g=$ $g^{-1}[\epsilon, g]+g^{-1} \delta g$ and the second component is the Maurer-Cartan 1-form on the group, i.e., the BRST ghost $v$.

The form

$$
\theta_{k-j, j}=\left.\int\left(g^{-1} \Delta g\right)^{k}\right|_{[j]}
$$

is closed in the $\delta$ cohomology complex for any $k=0,1,2, \ldots$ Here $\left.(\cdots)\right|_{[j]}$ denotes the component of $\delta$ degree (= ghost degree) $j$. In order that the integral is defined as a (graded) trace we have to assume that $k-j \geq p$.

Actually $\theta_{k-j, j}=0$ for all even $k$ 's. This follows from the anticommutator relations for $d, \delta$ and from the cyclic properties of the integral. The closedness for odd $k$ follows from

$$
\delta \theta=\int \delta\left(g^{-1} \Delta g\right)^{k}=\int \Delta\left(g^{-1} \Delta g\right)^{k}=-\int\left(g^{-1} \Delta g\right)^{k+1}=0,
$$

since $k+1$ is even.

For $j=k$ and with a proper normalization $\theta_{k, j}$ is the generator of $H^{k}\left(U_{p}^{\prime}, \mathbb{Z}\right)$ whereas for $j=0$ the integral gives the Fredholm index of $P_{+} g P_{+}$, where $P_{+}=$ $\frac{1}{2}(1+\epsilon)$.

The case $j=3$ is of interest to us. In this case $k-j=2 n$ is even, so $\theta_{2 n, 3}$ is an integral of an even $d$-form. We check that this is a nontrivial cohomology class. For that purpose, choose $H$ as the Hilbert space of square integrable sections in a tensor product of a Dirac spinor bundle over the torus $T^{2 n}=\left(S^{1}\right)^{2 n}$ and a trivial $\mathbb{C}^{N}$ bundle with the natural $U(N)$ action in the fibers. The gauge transformations $g: T^{2 n} \rightarrow U(N)$ act as multiplication operators on the spinor fields. Choosing $D_{0}$ as the Dirac operator defined by the metric on the torus and the trivial vector potential $A=0$ in the $\mathbb{C}^{N}$ bundle, one has, $[5,16]$,

$$
\operatorname{tr}_{C} \Gamma a_{0}\left[\epsilon, a_{1}\right] \ldots\left[\epsilon, a_{2 n}\right]=\frac{1}{n !(2 \pi i)^{n}} \int_{T^{2 n}} \operatorname{tr} a_{0} d a_{1} \ldots d a_{2 n}
$$

for smooth functions $a_{j}: T^{2 n} \rightarrow \mathbf{g}$, where $\mathbf{g}$ is the Lie algebra of $U(N)$ in its fundamental representation and ' $d$ ' on the right means the de Rham exterior derivative. It follows that, for any map $g(\cdot): S^{3} \rightarrow \operatorname{Map}\left(T^{2 n}, U(N)\right)$, we have

$$
\int_{S^{3}} \theta_{2 n, 3}=\frac{1}{n !(2 \pi i)^{n}} \int_{S^{3} \times T^{2 n}} \operatorname{tr}\left(g^{-1} d g\right)^{2 n+3}
$$


where $g$ is thought of as a $U(N)$ valued function on $S^{3} \times T^{2 n}$. In particular, for large $N$, this integral is equal to $24 \pi^{2} \times$ a nonzero integer when $g$ represents a nontrivial homotopy class in $\pi_{3+2 n}(U(N)) \simeq \mathbb{Z}$. Since $\theta_{2 n, 3}$ is nontrivial on the subgroup of smooth gauge transformations in $U_{2 n+}(H, \Gamma)$ it must represent a nonzero cohomology class of the latter group and therefore also in the moduli space $X=\mathcal{A} / \mathcal{G}_{0}$.

Specializing from the result of Theorem 3.1 (cf. Lemma A3 in the appendix) to the flat case $a=g^{-1}[\epsilon, g]$ we observe that the forms $\theta_{2 n, 3}$ can be written as sums of generalized traces of commutators plus a properly normalized 3-form $\operatorname{TR}\left(g^{-1} \delta g\right)^{3}$, modulo exact forms.

\section{SCHWINGER TERMS FROM THE DIXMIER-DOUADY CLASS}

In this section we shall work with the Fredholm modules based on the ideals $L_{p}$ instead of $L_{p+}$, and likewise with the groups of the type $U_{p}^{\prime}$. This is because we want to use available information (based on results in [13]) on the homotopy type of the infinite dimensional groups.

Let $\theta_{3}$ be a closed integral 3 -form on $X=\mathcal{A} / \mathcal{G}_{0}$. The pull back $\pi^{*} \theta_{3}=\psi_{3}$ with respect to the canonical projection is a closed 3 -form on the contractible space $\mathcal{A}$ and therefore $\psi_{3}=d \psi_{2}$ for some 2 -form $\psi_{2}$. (In this section $d$ means always the de Rham exterior derivative.)

Let $\mathcal{U}_{\alpha}$ be an open contractible subset of $X$ and write $\theta_{3}=d \theta_{2, \alpha}$ on $\mathcal{U}_{\alpha}$. We define

$$
\eta_{\alpha}=\psi_{2}-\pi^{*} \theta_{2, \alpha}
$$

on $\pi^{-1}\left(\mathcal{U}_{\alpha}\right)$. Now $d \eta_{\alpha}=\psi_{3}-d \pi^{*} \theta_{2, \alpha}=\psi_{3}-\pi^{*} d \theta_{2, \alpha}=\psi_{3}-\pi^{*} \theta_{3}=0$.

If $\mathcal{U}_{\beta}$ is another open subset of $X$ then on $\mathcal{U}_{\alpha} \cap \mathcal{U}_{\beta}$ we have $\eta_{\alpha}-\eta_{\beta}=\pi^{*}\left(\theta_{2, \alpha}-\right.$ $\left.\theta_{2, \beta}\right)$. From this follows, by the definition of the pull back map, that $\eta_{\alpha}$ and $\eta_{\beta}$ agree when evaluated along gauge orbits. Thus there is a vertical 2-form $\eta$ on $\mathcal{A}$, defined everywhere on $\mathcal{A}$, but which is closed only along gauge directions.

The vertical form $\eta$ defines an extension of the Lie algebra of $\mathcal{G}$ by the abelian Lie algebra a consisting of functions $f: \mathcal{A} \rightarrow \mathbb{C}$. The commutators are defined by

$$
[(X, f),(Y, g)]=\left([X, Y], \delta_{X} g-\delta_{Y} f+\eta(X, Y)\right)
$$

On the right the Lie algebra elements $X, Y$ are interpreted as vertical vector fields on $\mathcal{A}$. The Jacobi identity is precisely the condition that $\eta$ is closed along vertical directions.

We can compute $\eta$ more explicitly. Let $\mathcal{P} G$ denote the group of smooth paths in a group $G$ originating from the identity in $G$. The product is defined as a pointwise multiplication along paths. Any $A \in \mathcal{A}$ splits uniquely as $A=A_{0}+A_{1}$ to even and odd components with respect to $\Gamma$. The even component $A_{0}$ is equal to $i g(t)^{-1} \partial_{t} g(t)$ for a uniquely defined smooth function $g:[0,1] \rightarrow U_{p}^{\prime}(\Gamma, H)$ with $g(0)=1$. As in Section 4 , we can write $\mathcal{A}=\mathcal{A}_{1} \times \mathcal{P} U_{p}^{\prime}(\Gamma, H)$ and $\mathcal{A} / \mathcal{G}_{0}=\mathcal{A}_{1} \times U_{p}^{\prime}(\Gamma, H)$. 
Since $\mathcal{A}_{1}$ is a vector space, any closed form on $\mathcal{A}_{1}$ is exact and we may assume without loss of generality that $\theta_{3}$ is a pull-back of a form on $U_{p}^{\prime}(\Gamma, H)$ with respect to the projection on the second factor.

By a direct calculation one can check that $d \psi_{2}=\pi^{*} \theta_{3}$ when $\psi_{2}$ is defined as

$$
\psi_{2}(u, v)=\int_{0}^{1} \theta_{3}\left(g^{-1} \partial_{t} g, u_{0}(t), v_{0}(t)\right) d t,
$$

where the components $u_{0}, v_{0}$ of the tangent vectors $u, v \in \mathcal{A}$ are given as paths in the Lie algebra of $U_{p}^{\prime}(\Gamma, H)$. The along vertical directions, $\eta$ is equal to $\psi_{2}$ and thus

$$
\eta(g ; X, Y)=\int_{0}^{1} \theta_{3}\left(g^{-1} \partial_{t} g, X(t), Y(t)\right) d t,
$$

for periodic functions $X(t), Y(t)$ with values in $\operatorname{Lie}\left(U_{p}^{\prime}(\Gamma, H)\right)$.

Since $\pi_{2}\left(U_{p}^{\prime}(\Gamma, H)\right)=0$, the group $\mathcal{G}_{0}$ of (based) loops in $U_{p}^{\prime}(\Gamma, H)$ is simply connected. On the other hand, $\pi_{2}\left(\mathcal{L}_{k} U_{p}^{\prime}(\Gamma, H)\right)=\pi_{3}\left(U_{p}^{\prime}(\Gamma, H)\right)=\mathbb{Z}$ for any of the connected components $\mathcal{L}_{k}$ of the group $\mathcal{L} U_{p}^{\prime}$ of smooth loops in $U_{p}^{\prime}$. By Hurewicz' theorem, $H^{2}\left(\mathcal{L}_{k} U_{p}^{\prime}(\Gamma, H), \mathbb{Z}\right)=\mathbb{Z}$. In fact, the 2 -form $\eta$ restricted to a gauge orbit gives a representative for the basic class in $H^{2}\left(\mathcal{L}_{k} U_{p}^{\prime}, \mathbb{Z}\right)$.

We shall work only in the connected component of identity $\mathcal{L}_{0} U_{p}^{\prime}$. The group extension of $\mathcal{L}_{0} U_{p}^{\prime}(\Gamma, H)$ corresponding to the Lie algebra extension above can be explicitly constructed in the same way as was done in [17] in the case of groups of classical gauge transformations.

The construction starts from the Cartesian product $\mathcal{P}\left(\mathcal{L}_{0} U_{p}^{\prime}\right) \times \operatorname{Map}\left(\mathcal{A}, S^{1}\right)$. The product is defined as

$$
\left(f_{1}, \alpha_{1}\right)\left(f_{2}, \alpha_{2}\right)=\left(f_{1} f_{2}, \alpha_{1} \alpha_{2}^{f_{1}} \exp \left(2 \pi i \int_{K} \eta\right)\right.
$$

where $f_{1} f_{2}$ is just the point-wise product in the path group, $\alpha^{f}$ is the function obtained form $\alpha$ by gauge transforming (using the right action) the argument by $f(1) \in \mathcal{L}_{0} U_{p}^{\prime}$. The phase is evaluated by integrating the vertical 2 -form $\eta$ over a 2 -surface $K$ in the fiber at $A \in \mathcal{A}$. The surface is chosen such that its boundary consists of the paths $A \cdot f_{1}(t), A \cdot f_{1}(1) f_{2}(t)$, and $A \cdot f_{1}(t) f_{2}(t)$. The value of the phase factor does not depend on the choice of $K$ since the difference in the exponent is $2 \pi i$ times an integral of the integral 3 -form $\omega_{3}$ over a closed 3 -surface in $U_{p}^{\prime}$ and this is an integer.

The extension $\widehat{\mathcal{L}_{0} U_{p}^{\prime}}$ is now defined as

$$
\mathcal{P}\left(\mathcal{L}_{0} U_{p}^{\prime}\right) \times \operatorname{Map}\left(\mathcal{A}, S^{1}\right) / \mathcal{Q}
$$

where $\mathcal{Q}$ is the group of based loops (at the identity) in $\mathcal{L}_{0} U_{p}^{\prime}$ and the right action of $\mathcal{Q}$ is defined as the point-wise right action on $\mathcal{P}\left(\mathcal{L}_{0} U_{p}^{\prime}\right)$ and as the simultaneous phase shift $\alpha \rightarrow \alpha^{\prime}$,

$$
\alpha^{\prime}(A)=\alpha(A) \exp \left(2 \pi i \int_{\Sigma} \eta\right)
$$


where $\Sigma$ is the 2 -surface in the fiber through $A$, bounded by the loop $A \cdot g(t), g \in \mathcal{Q}$.

\section{THE CASE OF A NONCOMMUTATIVE TORUS}

In this section we want to apply the general results for computing cocycles on a noncommutative torus. We start by recalling the basic definitions, [18].

Consider an algebra consisting of finite linear combinations of 'noncommutative Fourier modes' $u^{r}$, where $r=\left(r_{1}, \ldots, r_{n}\right)$ is a vector of integers,

$$
u^{r}=e^{\pi i \sum_{j<k} \theta_{j k} r_{j} r_{k}} u_{1}^{r_{1}} \ldots u_{n}^{r_{n}}
$$

and the relations in the algebra are given as

$$
u_{i} u_{j}=e^{-2 \pi i \theta_{i j}} u_{j} u_{i},
$$

where $\theta$ is a real antisymmetric matrix. These relations lead to the multiplication formula

$$
u^{r} \cdot u^{r^{\prime}}=\lambda\left(r, r^{\prime}\right) u^{r+r^{\prime}}
$$

with $\lambda\left(r, r^{\prime}\right)=\exp \left(-\pi i \sum r_{j} \theta_{j k} r_{k}^{\prime}\right)$.

There is an antilinear conjugation defined by $\left(u^{r}\right)^{*}=u^{-r}$ and a trace $\tau$ such that $\tau\left(u^{r}\right)=0$ if $r \neq 0$ and $\tau\left(u^{0}\right)=\tau(1)=1$. We define a Hilbert space $H$ by tensoring the above algebra with the spinor and internal symmetry space $V=$ $\mathbb{C}^{[(n+1) / 2]} \otimes \mathbb{C}^{N}$ and completing with respect to the inner product defined by $<$ $u^{r}, u^{r^{\prime}}>=\tau\left(\left(u^{r}\right)^{*} u^{r^{\prime}}\right)=\delta_{r r^{\prime}}$. Here $[a]$ is the integer part of a real number $a$. The trace is extended to the tensor product of the torus algebra and of End $V$ by the matrix trace on End $V$. The adjoint of a multiplication operator by the element $u^{r}$ is $\left(u^{r}\right)^{*}=u^{-r}$.

There is a commutative family of derivations $\delta_{j}(j=1,2, \ldots, n)$ defined by $\delta_{j}\left(u^{r}\right)=r_{j} u^{r}$. These derivations are represented by unbounded operators $P_{j}$ in the Hilbert space $H$ through $P_{j} u^{r}=r_{j} u^{r}$ which means that $\left[P_{j}, u^{r}\right]=r_{j} u^{r}$ for the corresponding multiplication operators $u^{r}$ (we use the same notation for elements in the noncommutative torus algebra and vectors in the Hilbert space).

The (free) Dirac operator is defined by

$$
D_{0}=\sum \gamma^{k} \delta_{k},
$$

where the gamma matrix algebra is defined using the standard Euclidean metric, $\gamma_{i} \gamma_{j}+\gamma_{j} \gamma_{i}=2 \delta_{i, j}$.

As in the case of pseudodifferential operators on an ordinary torus we can introduce a symbol calculus. Consider first classical PSDO symbols $f=f(p)$ which are functions of the momenta $p_{j}$ only and which have an asymptotic expansion 
$f \sim f_{N}+f_{N-1}+\ldots$ as a series of homogenenous symbols $f_{j}$ of order $j$ in the momenta, i.e, $f_{j}(\lambda p)=\lambda^{j} f(p)$ for $\lambda>1$ and for large momenta. Each such a symbol defines an (in general unbounded) operator in $H$ through $f u^{r}=f(r) u^{r}$. More generally, we consider operators

$$
f=\sum_{r} u^{r} f^{(r)}
$$

where each $f^{(r)}$ is a classical symbol of the above type tensored with a matrix operating in $V$. The definition makes sense even for infinite linear combinations assuming that the sequence $\left|f^{(r)}(p)\right|$ is rapidly decreasing as $|r| \rightarrow \infty$ for all values of $p$. From the definitions follows that

$$
u^{-r} f(p) u^{r}=f(p+r)
$$

and therefore the product of a pair $f, g$ of operators is defined by the 'star product'

$$
(f * g)^{(r)}(p)=\sum_{s} f^{(r-s)}(p) g^{(s)}(p+s) \lambda(r-s, s) .
$$

The only difference as compared to the commutative torus is the appearance of complex phases $\lambda$ on the right-hand side. The trace of the operator $f$ (when it is defined) is given by

$$
\operatorname{tr} f=\sum_{p \in \mathbb{Z}^{n}} \operatorname{tr} f^{(0)}(p),
$$

where the trace on the right is the ordinary matrix trace in $V$. As usual, $f$ is traceclass if and only if $\operatorname{ord}(f)<-n$. The border line case ord $(f)=-n$ is of special interest to us.

For complex numbers $z$ with an enough large real part one can define

$$
\zeta(z ; f)=\operatorname{tr} f(|P|+1)^{-z}
$$

If $f$ is trace-class then $\zeta$ is holomorphic at $z=0$ and $\operatorname{tr} f=\zeta(0 ; f)$. If $\operatorname{ord}(f) \geq-n$, the function $\zeta$ has in general a pole at $z=0$. The pole depends only on the component $f_{-n}$. The proof of this statement follows from the corresponding result for PSDO's on the commutative torus since the spectrum of $f^{(0)}$ is exactly the same as in the commutative case. For the same reason the the residue at $z=0$ can be calculated as a momentum space integral,

$$
\operatorname{Res}(f)=\operatorname{res}_{z=0} \zeta(z ; f)=\int_{|p|=1} \operatorname{tr} f_{-n}^{(0)}(p) d p
$$

For classical PSDO's on a compact manifold there is a useful formula relating the renormalized (noncyclic) trace of a commutator to an operator residue. This 
formula was used for calculation of Schwinger terms in $[8,19]$ and later generalized in [20] to a wider class of operators. First one defines a renormalized trace for nontraceclass PSDO's by

$$
\operatorname{TR} A=\lim _{z \rightarrow 0}\left(\operatorname{tr} A|D|^{-z}-\frac{1}{z} \operatorname{Res} A\right) .
$$

The lack of cyclicity of TR is given by

$$
\operatorname{TR}[A, B]=\operatorname{Res} A[\ell, B],
$$

where $\ell=\log \left|D_{0}\right|=\log |P|$. We check that the same formula holds in the case of noncommutative torus. By linearity, it is sufficient to prove the formula for each Fourier mode separately. So we take $A=u^{r} f(p)$ and $B=u^{s} g(p)$. By the definition of the trace, TR $A B$ is nonzero only when $s=-r$, so we assume this. But now $\operatorname{TR} A B=\operatorname{TR} \lambda(r, s) \tilde{f} g$, where $\tilde{f}(p)=f(p-r)$. Since $\lambda(r, s)=1$ when $s=-r$, the trace formula for $\mathrm{NC}$ torus reduces to the formula on commutative torus and thus (7.8) holds.

Let us look closer at the case $n=3$. We have seen that the Schwinger term in three dimensions is equivalent (modulo coboundaries) to the 'local' expression

$$
8 \omega_{3,2}=\operatorname{TR}\{[X, \epsilon a[\epsilon, Y]]-[Y, \epsilon a[\epsilon, X]]-2[X \epsilon, Y]+2[Y \epsilon, X]\} .
$$

We now apply the residue formula in the case of noncommutative multiplication operators $X, Y$ and we obtain

$$
8 \omega_{3,2}=-\operatorname{Res} \epsilon a[\epsilon, Y][\ell, X]+\operatorname{Res} \epsilon a[\epsilon, X][\ell, Y] .
$$

Taking $a=F-\epsilon$ and $F=\left(D_{0}+A\right)\left|D_{0}+A\right|^{-1}$, the sign of a Dirac operator defined by a potential $A=\sigma^{k} A_{k}$ on the noncommutative torus, a straight-forward computation gives

$$
\omega_{3,2}=\frac{i \pi}{6} \tau\left(A\left[\left[D_{0}, X\right],\left[D_{0}, Y\right]\right]\right)=\frac{i \pi}{3} \epsilon^{i j k} \tau\left(A_{i}\left(\delta_{j}(X) \delta_{k}(Y)-\delta_{j}(Y) \delta_{k}(X)\right)\right) .
$$

We have used the expansion

$$
F=\epsilon+A /|P|-\epsilon A^{k} P_{k} /|P|^{2}+O\left(1 /|P|^{2}\right)
$$

and the formulas

$$
\begin{aligned}
{\left[\epsilon, u^{(r)}\right] } & =u^{(r)}\left(u^{(-r)} \epsilon u^{(r)}-\epsilon\right)=u^{(r)}\left(\frac{\left(P_{k}+r_{k}\right) \sigma^{k}}{|P+r|}-\frac{P_{k} \sigma^{k}}{|P|}\right) \\
& =u^{(r)}\left(r_{k} \sigma^{k} /|P|-\epsilon P_{k} r^{k} /|P|^{2}+O\left(1 /|P|^{2}\right)\right)
\end{aligned}
$$

and similarly

$$
\left[\ell, u^{(r)}\right]=u^{(r)} P_{k} r^{k} /|P|^{2}+O\left(1 /|P|^{2}\right) .
$$

The derivation of (7.10) is then completed by taking account

$$
\operatorname{tr} \sigma_{i} \sigma_{j} \sigma_{k}=2 i \epsilon_{i j k} \text { and } \operatorname{Res} \frac{p_{i} p_{j}}{|p|^{5}}=\frac{4 \pi}{3} \delta_{i, j} .
$$




\section{APPENDIX: NONCOMMUTATIVE DESCENT EQUATIONS}

We assume linear operators $d$ and $\delta$ acting on polynomials generated by $a, v, d(a)$, $d(v), \delta(a)$, and $\delta(v)$, such that $d^{2}=0=\delta^{2}=d \delta+\delta d$. These operations are then uniquely fixed by the additional rules in (2.6) together with $\epsilon^{2}=I$. As mentioned in the text, we will use the simplified notation $x=a, \epsilon, v$ and $\delta$ short for $s(x)$ defined in (2.7) resp. (2.8) for even resp. odd Fredholm modules throughout this Appendix except in the Remark at the end.

We now state and give a proof of the non-commutative descent equations. We define

$$
\begin{aligned}
F(t) & =t d(a)+t^{2} a^{2}+(1-t) d(v) \\
F^{\prime}(t) & =\left(t^{2}-t\right) v^{2}+t d(v)
\end{aligned}
$$

and

$$
\omega_{2 n-1}=\int_{0}^{1} d t \Psi_{2 n-1}(t), \quad \tilde{\omega}_{2 n-1}=\int_{0}^{1} d t t \Psi_{2 n-1}(t),
$$

and similarly for $\omega_{2 n-1}^{\prime}$ and $\tilde{\omega}_{2 n-1}^{\prime}$, where

$$
\begin{aligned}
& \Psi_{2 n-1}(t)=\sum_{\nu=0}^{n-1} F(t)^{n-1-\nu} a F(t)^{\nu} \\
& \Psi_{2 n-1}^{\prime}(t)=\sum_{\nu=0}^{n-1} F^{\prime}(t)^{n-1-\nu} v F^{\prime}(t)^{\nu} .
\end{aligned}
$$

The non-commutative descent equations can be summarized by the following algebraic identities:

\section{Lemma A1.}

$$
\begin{aligned}
& \delta\left(\omega_{2 n-1}\right)+d\left(\omega_{2 n-1}\right)=-\left[v, \omega_{2 n-1}\right]_{+}-\left[a, \tilde{\omega}_{2 n-1}\right]_{+}+F^{n}-(d(v))^{n} \\
& \delta\left(\omega_{2 n-1}^{\prime}\right)+d\left(\omega_{2 n-1}^{\prime}\right)=-\left[v, \tilde{\omega}_{2 n-1}^{\prime}\right]_{+}+(d(v))^{n}
\end{aligned}
$$

where $F=d(a)+a^{2}$.

Comparing equal powers of $v$ we obtain from (A.2)

$$
\begin{aligned}
d\left(\omega_{2 n-1,0}\right)=-\left[a, \tilde{\omega}_{2 n-1,0}\right]_{+}+F^{n} \\
\delta\left(\omega_{2 n-k, k-1}\right)+d\left(\omega_{2 n-1-k, k}\right)=-\left[v, \omega_{2 n-k, k-1}\right]_{+}-\left[a, \tilde{\omega}_{2 n-k-1, k}\right]_{+} \\
k=1, \ldots n-1 \\
\delta\left(\omega_{n, n-1}\right)+d\left(\omega_{n-1, n}\right)=-\left[v, \omega_{n, n-1}\right]_{+} \\
\delta\left(\omega_{2 n-k, k-1}\right)+d\left(\omega_{2 n-k-1, k}\right)=-\left[v, \tilde{\omega}_{2 n-k, k-1}\right]_{+} \\
k=n+1, \ldots 2 n-1 \\
\delta\left(\omega_{0,2 n-1}\right)=-\left[v, \tilde{\omega}_{0,2 n-1}\right]_{+}
\end{aligned}
$$


where the $k$ in $\omega_{2 n-k-1, k}$ is the ghost degree i.e. power in $v$, and

$$
\begin{aligned}
& \omega_{2 n-1}=\omega_{2 n-1,0}+\omega_{2 n-2,1}+\ldots+\omega_{n, n-1}+\ldots \\
& \omega_{2 n-1}^{\prime}=\omega_{n-1, n}+\omega_{n-2, n+1}+\ldots+\omega_{0,2 n-1}+\ldots
\end{aligned}
$$

(note that the equation for $k=n$ is obtained by combining the two equations

$$
\begin{aligned}
& \delta\left(\omega_{n, n-1}\right)=-\left[v, \omega_{n, n-1}\right]_{+}-(d(v))^{n} \\
& d\left(\omega_{n-1, n}\right)=(d(v))^{n}
\end{aligned}
$$

obtained from the first and the second relation in Eq. (A.2), respectively).

Proof of Eq. (A.2). We observe that

$$
F(t)=(\epsilon+\delta+t a+v)^{2}-I
$$

implying $\partial_{t}(F(t))=[\epsilon+\delta+t a+v, a]_{+}$, and therefore

$$
\begin{aligned}
F^{n}-(d(v))^{n} & =\int_{0}^{1} d t \partial_{t}(F(t))^{n}= \\
& \int_{0}^{1} d t \sum_{\nu=0}^{n-1} F(t)^{n-1-\nu}[\epsilon+\delta+t a+v, a]_{+} F(t)^{\nu}= \\
& \int_{0}^{1} d t\left[\epsilon+\delta+t a+v, \Psi_{2 n-1}\right]_{+}
\end{aligned}
$$

(we used that $\epsilon+\delta+t a+v$ commutes with $F(t)$ ), implying the first identity in Eq. (A.2). In a similar manner,

$$
F^{\prime}(t)=(\epsilon+\delta+t v)^{2}-I
$$

implies $\partial_{t}\left(F^{\prime}(t)\right)=[\epsilon+\delta+t v, v]_{+}$and thus

$$
\begin{aligned}
(d(v))^{n} & =\int_{0}^{1} d t \partial_{t}\left(F^{\prime}(t)\right)^{n}= \\
& \int_{0}^{1} d t \sum_{\nu=0}^{n-1} F^{\prime}(t)^{n-1-\nu}[\epsilon+\delta+t v, v]_{+} F^{\prime}(t)^{\nu}= \\
& \int_{0}^{1} d t\left[\epsilon+\delta+t v, \Psi_{2 n-1}^{\prime}\right]_{+}
\end{aligned}
$$

yielding the second identity in Eq. (A.2).

Some applications. A simple special case of Eqs. (A.3) is the following result. We point out that here, $x=a$ and $\epsilon$ need not to be interpreted as $s(x)$ but can be taken directly as operators in $H$. 
Lemma A2. For flat a, i.e., $a=g^{-1}[\epsilon, g]=\left[g^{-1} \epsilon, g\right]$ for some invertible operator $g$, the following holds true

$$
a^{2 n+1}=4 a^{2 n-1}+2\left[\epsilon, a^{2 n-1} \epsilon\right]-\left[a, \epsilon a^{2 n-1}\right]
$$

for all positive integers $n$.

Proof. For flat $a$ we have $F=d(a)+a^{2}=0$, and thus the first equation in (A.3), implies $\left[\epsilon, \omega_{2 n-1,0}\right]_{+}=-\left[a, \tilde{\omega}_{2 n-1,0}\right]_{+}$where $\omega_{2 n-1,0}$ is obtained from $\omega_{2 n-1}$ above by substituting $F(t)=\left(t^{2}-t\right) a^{2}$, i.e., $\omega_{2 n-1,0}=\int_{0}^{1} d t n\left(t^{2}-t\right)^{n-1} a^{2 n-1} \equiv c_{n} a^{2 n-1}$, and similarly $\tilde{\omega}_{2 n-1,0}=\int_{0}^{1} d t \operatorname{tn}\left(t^{2}-t\right)^{n-1} a^{2 n-1} \equiv \tilde{c}_{n} a^{2 n-1}$. Note that

$$
2 \tilde{c}_{n}-c_{n}=\int_{0}^{1} d t n(2 t-1)\left(t^{2}-t\right)^{n-1}=0
$$

implying

$$
2\left[\epsilon, a^{2 n-1}\right]_{+}=-\left[a, a^{2 n-1}\right]_{+}
$$

Multiplying this identity by $\epsilon$ we obtain

$$
\begin{aligned}
2 \epsilon\left[\epsilon, a^{2 n-1}\right]_{+} & =4 a^{2 n-1}+2\left[\epsilon, a^{2 n-1} \epsilon\right]= \\
-\epsilon\left[a, a^{2 n-1}\right]_{+} & =-[\epsilon, a]_{+} a^{2 n-1}+\left[a, \epsilon a^{2 n-1}\right] .
\end{aligned}
$$

Inserting $[\epsilon, a]_{+}=d(a)=-a^{2}$ we obtain Eq. (A.4).

Note that the Lemma gives as a byproduct a simple proof of the known index formula for the Fredholm index ind $P_{+} g P_{+}$, where $g \in U_{p}^{\prime}\left(H_{+} \oplus H_{-}\right)$and $P_{+}$is the projection to $H_{+}$. Namely, since according to [13] the connected components of $U_{p}^{\prime}$ are labelled by the Fredholm index of $P_{+} g P_{+}$, the index is a homotopy invariant, and the multiplication operator by the function $f(x)=e^{i n x}$ on the unit circle $0 \leq x \leq 2 \pi$ has index ind $P_{+} f P_{+}=n$ (by a simple exercise in Fourier analysis), it is sufficient to check that

$$
n=\frac{1}{2} \operatorname{tr} f^{-1}[\epsilon, f]
$$

and the general formula

$$
\operatorname{ind} P_{+} g P_{+}=2^{-p} \operatorname{tr}\left(g^{-1}[\epsilon, g]\right)^{p}
$$

follows for odd positive integers $p$.

Local cocyles. The basic result implying the existence of the local cocycles is the following 
Lemma A3. The forms defined above obey

$$
\begin{aligned}
\epsilon\left[v, \omega_{2 n-k, k-1}\right]_{+}+\epsilon\left[a, \tilde{\omega}_{2 n-k-1, k}\right]_{+} & \simeq c_{n, k} \omega_{2 n-k+1, k}, \quad k=1, \ldots, n-1 \\
\epsilon\left[v, \omega_{n, n-1}\right]_{+} & \simeq c_{n, n} \omega_{n+1, n} \\
\epsilon\left[v, \tilde{\omega}_{2 n-k, k-1}\right]_{+} & \simeq c_{n, k} \omega_{2 n-k+1, k}, \quad k=n+1, \ldots, 2 n
\end{aligned}
$$

where

$$
c_{n, k}=\frac{2 n+1-k}{n+1}
$$

and ' $\simeq$ ' means 'equal up to commutator terms'.

Proof. To prove the first relation in Eq. (A.5) we observe

$$
\begin{aligned}
\epsilon\left[v, \omega_{2 n-k, k-1}\right]_{+}+\epsilon\left[a, \tilde{\omega}_{2 n-k-1, k}\right]_{+} & =[\epsilon, v]_{+} \omega_{2 n-k, k-1}+[\epsilon, a]_{+} \tilde{\omega}_{2 n-k-1, k}+ \\
{\left[\epsilon \omega_{2 n-k, k-1}, v\right]+\left[\epsilon \tilde{\omega}_{2 n-k-1, k}, a\right] } & \simeq d(v) \omega_{2 n-k, k-1}+d(a) \tilde{\omega}_{2 n-k-1, k}
\end{aligned}
$$

and thus what we actually need to prove is

$$
d(v) \omega_{2 n-k, k-1}+d(a) \tilde{\omega}_{2 n-k-1, k} \simeq \frac{2 n+1-k}{n+1} \omega_{2 n-k+1, k}
$$

For that it is convenient to define an ordering symbol \{\} so that

$$
\sum_{N=0}^{\infty}\left(a_{1}+a_{2}+\ldots a_{k}\right)^{N}:=\sum_{m_{1}, m_{2}, \ldots, m_{k}=0}^{\infty}\left\{a_{1}^{m_{1}}, a_{2}^{m_{2}}, \ldots, a_{k}^{m_{k}}\right\}
$$

for all operators $a_{j}$ (we regard this as generating function defining all possible $\left.\left\{a_{1}^{m_{1}}, a_{2}^{m_{2}}, \ldots, a_{k}^{m_{k}}\right\}\right)$. We observe that this definition implies

$$
a_{1}\left\{a_{1}^{m_{1}}, a_{2}^{m_{2}}, \ldots, a_{k}^{m_{k}}\right\} \simeq \frac{m_{1}+1}{m_{1}+m_{2}+\ldots+m_{k}+1}\left\{a_{1}^{m_{1}+1}, a_{2}^{m_{2}}, \ldots, a_{k}^{m_{k}}\right\} .
$$

We can thus write $\omega_{2 n-k-1, k}=\int_{0}^{1} d t \Omega_{2 n-k-1, k}$ and $\tilde{\omega}_{2 n-k-1, k}=\int_{0}^{1} d t t \Omega_{2 n-k-1, k}$ where

$$
\Omega_{2 n-k-1, k}=\left\{u^{k},(p+q)^{n-k-1}, a\right\}=\sum_{\ell=0}^{n-k-1}\left\{u^{k}, p^{n-k-\ell-1}, q^{\ell}, a\right\}
$$

with $u \equiv(1-t) d(v), p \equiv t d(a)$ and $q \equiv t^{2} a^{2}$. To prove Eq. (A.6) we thus need to show that

$$
\begin{gathered}
\frac{2 n+1-k}{n+1} \int_{0}^{1} d t\left\{u^{k}, p^{n-k-\ell}, q^{\ell}, a\right\} \simeq \\
\int_{0}^{1} d t\left(\frac{u}{1-t}\left\{u^{k-1}, p^{n-k-\ell}, q^{\ell}, a\right\}+p\left\{u^{k}, p^{n-k-\ell-1}, q^{\ell}, a\right\}\right),
\end{gathered}
$$


which follows from

$$
\begin{array}{r}
\int_{0}^{1} d t\left(\frac{2 n+1-k}{n+1}-\frac{k}{(n+1)(1-t)}-\frac{n-k-\ell}{n+1}\right) t^{n-k-\ell} t^{2 \ell}(1-t)^{k} \\
=\frac{1}{n+1} \int_{0}^{1} d t \partial_{t}\left(t^{n+1-k+\ell}(1-t)^{k}\right)=0 .
\end{array}
$$

The proof of the second and third relations in Eq. (A.5) is similar but simpler: Recalling $u=(1-t) d(v)$ we get

$$
\begin{aligned}
& \epsilon\left[v, \omega_{n, n-1}\right]_{+} \simeq d(v) \omega_{n, n-1}=\int_{0}^{1} d t \frac{1}{1-t} u\left[u^{n-1}, a\right]_{+}= \\
& \int_{0}^{1} d t \frac{1}{1-t} \frac{n}{n+1}\left[u^{n}, a\right]_{+}=\int_{0}^{1} d t\left[u^{n}, a\right]_{+}=c_{n, n} \omega_{n+1, n}
\end{aligned}
$$

since

$$
\frac{n}{n+1} \int_{0}^{1} d t(1-t)^{n-1}=\int_{0}^{1} d t(1-t)^{n},
$$

and finally, with $r \equiv t d(v)$ and $s \equiv\left(t^{2}-t\right) v^{2}$

$$
\begin{array}{r}
\epsilon\left[v, \tilde{\omega}_{2 n-k, k-1}\right]_{+} \simeq d(v) \tilde{\omega}_{2 n-k, k-1}=\int_{0}^{1} d t r\left\{r^{2 n-k}, s^{k-1-n}, v\right\} \simeq \\
\int_{0}^{1} d t \frac{2 n+1-k}{n+1}\left\{r^{2 n+1-k}, s^{k-1-n}, v\right\}=c_{n, k} \omega_{2 n-k+1, k} .
\end{array}
$$

We obtain a generalization of Lemma A2:

Theorem A4. Let

$$
\omega_{2 n-1-k, k}^{\prime}=C_{n, k} \omega_{2 n-1-k, k} \quad \text { where } \quad C_{n, k}=(-1)^{n} \frac{(2 n-1-k) ! !}{2^{n} n !} .
$$

Then

$$
\omega_{2 n+1-k, k}^{\prime} \simeq \omega_{2 n-1-k, k}^{\prime}-\frac{C_{n, k}}{2 C_{n, k-1}} \delta\left(\epsilon \omega_{2 n-k, k-1}^{\prime}\right)
$$

Proof. Using the Lemmas above, we observe that

$$
-\delta\left(\epsilon \omega_{2 n-k, k-1}\right)+2 \omega_{2 n-1-k, k} \simeq-c_{n, k} \omega_{2 n+1-k, k} \quad k=1,2, \ldots, 2 n-1,
$$

where we have used that $\epsilon d(\omega)=\epsilon[\epsilon, \omega]_{+} \simeq 2 \omega$. By multiplying this with $(1 / 2) C_{n, k}$ and using the recursion relation

$$
-\frac{1}{2} c_{n, k} C_{n, k}=C_{n+1, k}
$$

we get Eq. (A.7). 
Theorem A5. For even $k$ the $k$-cocyle

$$
\omega_{2 n+1-k, k}^{l o c}=\omega_{2 n+1-k, k}+\delta B_{2 n-k, k-1}
$$

is local for some cochain $B_{2 n-k, k-1}$, i.e., a sum of commutator terms. In the case when $k$ is odd one has to add a term proportional to $\omega_{0, k}$ to the right-hand-side of the equation.

Proof. Eq. (A.8) implies

$$
\begin{aligned}
\omega_{2 n+1-k, k} & \simeq-\frac{2}{c_{n, k}} \omega_{2 n-1-k, k}-\frac{1}{c_{n, k}} \delta\left(\omega_{2 n-k, k-1}\right) \simeq \\
& \sum_{\ell^{\prime}=0}^{\ell}(-1)^{\ell^{\prime}+1} \frac{2^{\ell^{\prime}}}{c_{n, k} c_{n-1, k} \cdots c_{n-\ell^{\prime}, k}} \delta\left(\omega_{2 n-2 \ell^{\prime}-k, k-1}\right) \\
& +(-1)^{\ell+1} \frac{2^{\ell}}{c_{n, k} c_{n-1, k} \cdots c_{n-\ell, k}} \omega_{2 n-1-2 \ell-k, k} .
\end{aligned}
$$

In particular,

$$
\omega_{2 n+1-2 k, 2 k} \simeq \sum_{\ell^{\prime}=0}^{n-k}(-1)^{\ell^{\prime}+1} \frac{1}{2} \frac{(n+1) !\left(n-2 k-\ell^{\prime}\right) !}{\left(n-\ell^{\prime}\right) !(n-2 k) !} \delta\left(\omega_{2 n-2 \ell^{\prime}-2 k, 2 k-1}\right)
$$

and

$$
\begin{aligned}
\omega_{2 n-2 k, 2 k+1} & \simeq \sum_{\ell^{\prime}=0}^{n-k-1}(-1)^{\ell^{\prime}+1} \frac{1}{2} \frac{(n+1) !\left(n-2 k-\ell^{\prime}-1\right) !}{\left(n-\ell^{\prime}\right) !(n-2 k-1) !} \delta\left(\omega_{2 n-2 \ell^{\prime}-2 k-1,2 k}\right) \\
& +(-1)^{n-k} \frac{1}{2}\left(\begin{array}{c}
n+1 \\
k
\end{array}\right) \omega_{0,2 k+1}
\end{aligned}
$$

which also gives an explicit formula for $B_{2 n-k, k-1}$.

Remark: Theorem A5 does not yet imply Theorem 3.1: we recall that we worked on an abstract BRST level here and $x=a, \epsilon, v$ and $\delta$ etc. was short for $s(x)$ as in Eq. (2.7) resp. (2.8). As discussed in the text, to obtain forms $\omega_{2 n-1-k, k}\left(a ; X_{1}, \ldots, X_{k}\right)$ one has to evaluate the ghosts $v$ at tangent vectors $X_{j}$ and, in the case of an odd Fredholm module, take the trace in the auxiliary space with the Pauli matrix $\sigma_{3}$. To complete the proof of Theorem 3.1 one needs to check that the commutators mentioned in Theorem A5 - which are on the abstract BRST level - turn into the commutators on the operator level mentioned in Theorem 3.1. We now show that this is indeed the case. For that we need to distinguish $s(x)$ from $x$. The 
commutators referred to in Theorem A5 are then of the form $[s(\alpha), s(\beta)]$ where $s(\alpha)$ and $s(\beta)$ are monomials in $s(v), s(a)$ and $s(\epsilon)$.

In the case of an even Fredholm module, there is no extra auxiliary space (cf. $(2.7)$ ), and the BRST commutators $[s(\alpha), s(\beta)]$ immediately become operator commutator after the evaluation of the ghosts at the tangent vectors.

We thus can concentrate on odd Fredholm modules. Let $n_{\alpha}$ be the ghost degree of $\alpha$ (the number of $s(v)$ ) and $d_{\alpha}$ the sum of the numbers of $s(\epsilon)$ and $s(a)$ (which is of course only defined modulo 2), and similarly for $\beta$. Then $s(\alpha)= \pm \alpha \otimes \sigma_{3}^{d_{\alpha}} \sigma_{1}^{n_{\alpha}}$ and similarly for $\beta$, and thus

$$
[s(\alpha), s(\beta)]= \pm\left((-1)^{n_{\alpha} d_{\beta}} \alpha \beta-(-1)^{d_{\alpha} n_{\beta}} \beta \alpha\right) \otimes \sigma_{3}^{d_{\alpha}+d_{\beta}} \sigma_{1}^{n_{\alpha}+n_{\beta}} .
$$

In the odd case the ghost degree $k=n_{\alpha}+n_{\beta}$ is even and $d_{\alpha}+d_{\beta}$ is odd. Evaluating $[s(\alpha), s(\beta)]$ for Lie algebra elements $X_{j}$, we obtain (up to an irrelevant overall factor)

$$
\begin{aligned}
& \sum_{P \in S_{k}} \operatorname{sign}_{P}\left(\alpha\left(X_{P(1)}, \ldots, X_{P\left(n_{\alpha}\right)}\right) \beta\left(X_{P\left(n_{\alpha}+1\right)}, \ldots, X_{P(k)}\right)-\right. \\
&\left.(-1)^{n_{\alpha} n_{\beta}}(-1)^{n_{\alpha} d_{\beta}+d_{\alpha} n_{\beta}} \beta\left(X_{P\left(n_{\alpha}+1\right)}, \ldots, X_{P(k)}\right) \alpha\left(X_{P(1)}, \ldots, X_{P\left(n_{\alpha}\right)}\right)\right)
\end{aligned}
$$

where $S_{k}$ is the permutation group of $k$ elements and sign $n_{P}$ gives the parity of a permutation $P$. One now needs to show that this always corresponds to a sum of commutators. For that we need only to check that the exponent $n_{\alpha} n_{\beta}+n_{\alpha} d_{\beta}+n_{\beta} d_{\alpha}$ is always even. But this follows from the fact that here $n_{\alpha}+n_{\beta}$ is even and $d_{\alpha}+d_{\beta}$ is odd.

\section{REFERENCES}

[1] J. Schwinger: Field theory commutators. Phys. Rev. Lett. 3, 296-297 (1959). S. Adler: Axial vertex in spinor electrodynamics. Phys. Rev. 177, 2426 (1969). J. Bell and R. Jackiw: A PCAC puzzle: $\pi_{0} \rightarrow \gamma \gamma$ in the sigma model. Nuevo Cimento, 60A, 47 (1969).

[2] J. Mickelsson: Chiral anomalies in even and odd dimensions. Commun. Math. Phys. 97, 361 (1985). L.D. Faddeev and S. Shatashvili: Algebraic and Hamiltonian methods in the theory of nonabelian anomalies. Theoret. Math. Phys. 60, 770 (1985)

[3] K. Fujikawa: Path integral for gauge theories with fermions. Phys. Rev. D (3), 21, no 10, 2848-2858 (1980). M.F. Atiyah and I. Singer: Dirac operators coupled to vector potentials. Proc. Natl. Acad. Sci. (USA) 81, no. 8, 2597-2600 (1984)

[4] J.M. Gracia-Bondia and J.C.Varilly: On the ultraviolet behavior of quantum fields over noncommutative manifolds. Internat. J. of Modern. Phys. A 14, no. 8, 1305-1323 (1999) 
[5] A. Connes: Noncommutative Geomerty. Academic Press, San Diego (1994)

[6] E. Langmann: Descent equations of Yang-Mills anomalies in noncommutative geometry. J. Geom. Phys. 22, no. 3, 259-279 (1997)

[7] L. Bonora, M. Schnabl, and A. Tomasiello: A note on consistent anomalies in noncommutative Y-M theories. Phys. Lett. B485, 311-313 (2000). J.M. GraciaBondia and C.P. Martin: Chiral gauge anomalies on noncommutative $\mathbb{R}^{4}$. Phys. Lett. B479, 321-328 (2000)

[8] J. Mickelsson: Wodzicki residue and anomalies of current algebras. In: Integrable Models and Strings. Springer Lecture Notes in Physics 436, 123-135, (1994)

[9] E. Langmann and J. Mickelsson: Elementary derivation of the chiral anomaly. Lett. Math. Phys. 36, no. 1, 45-54 (1996)

[10] A. Carey, M. Murray, and J. Mickelsson: Bundle gerbes applied to field theory. Revs. Math. Phys. 12, no. 1, 65-90 (2000)

[11] H. Araki: Bogoliubov automorphisms and Fock representations of the algebra of canonical anticommutation relations. Contemp. Math. 62, 23-141, Amer. Math. Soc., Providence, RI (1987)

[12] B. Zumino: Chiral anomalies and differential geometry. Relativity, Groups, and Topology, II (Les Houches 1983), notes prepared by K. Sibold, 1291-1322. North-Holland, Amsterdam and New York (1984)

[13] R. Palais: On the homotopy type of certain groups of operators. Topology 3, 271-279 (1965)

[14] J. Dixmier and A. Douady: Champs continus d'espaces hilbertiens et de $C^{*}$ algebres. Bull. Soc. Math. Fr. 91, 227-284 (1963)

[15] A. Carey and J. Mickelsson: A gerbe obstruction to quantization of fermions on odd-dimensional manifolds with boundary. Lett. Math. Phys. 51, 145-160 (2000)

[16] E. Langmann: Noncommutative integration calculus. J. Math. Phys. 36, no. $7,3822-3825$ (1995)

[17] J. Mickelsson: Kac-Moody groups, topology of the Dirac determinant bundle, and fermionization. Commun. Math. Phys. 110, no.2, 173-183 (1987)

[18] M. Rieffel: Noncommutative tori - a case study of noncommutative differentiable manifolds. Contemp. Math. 105, 191-211. Amer. Math. Soc., Providence, RI (1990)

[19] M. Cederwall, G. Ferretti, B.E.W. Nilsson, A. Westerberg: Schwinger terms and cohomology of pseudodifferential operators. Commun. Math. Phys. 175, no.1, 203-220 (1996)

[20] S. Paycha: Renormalized traces as a looking glass into infinite dimensional geometry. Preprint no. 671, Sonderforschungsbereich 256, Bonn (2000). A. Cardona, C. Ducourtioux, J.P. Magnot, and S. Paycha: Weighted traces on the algebra of pseudodifferential operators and geometry of loop groups. math.OA/0001117

[21] L.-E. Lundberg: Quasi-free "second quantization". Commun. Math. Phys. 50, no.2, 103-112 (1976)

[22] J. Mickelsson: Current Algebras and Groups. Plenum Press, London and New York (1989) 
[23] R. Bhatia: Matrix Analysis. Graduate Texts in Mathematics 169, SpringerVerlag, New York (1997)

[24] J.M. Gracia-Bondia, J.C. Varilly, and H. Figueroa: Elements of Noncommutative Geometry. Birkhäuser, Boston (2001) 